\title{
A liquid +TIP-network regulates microtubule dynamics through tubulin condensation
}

\author{
Julie Miesch ${ }^{1 \#}$, Robert T. Wimbish ${ }^{1 \#}$, Marie-Claire Velluz ${ }^{1}$, Charlotte Aumeier ${ }^{1,2 *}$
}

${ }^{1}$ Department of Biochemistry, University of Geneva, 1211 Geneva, Switzerland

${ }^{2}$ National Center for Competence in Research Chemical Biology, University of Geneva, 1211 Geneva, Switzerland

\# equal contribution

*Correspondence to: $\underline{\text { Charlotte.aumeier@unige.ch }}$

\section{SUMMARY}

Regulation of microtubule dynamics is required for many biological processes. Here we uncovered a novel mechanism to regulate microtubule dynamics: local tubulin condensation. We show that two microtubule tip-binding proteins, CLIP-170 and EB3, undergo phase separation and form a liquid-like network at growing microtubule tips. In vitro and in cells this liquid network can condense tubulin. This process of tubulin co-condensation is spatially initiated at the microtubule tip and temporally regulated to occur only when microtubules grow. Tubulin condensation at the growing microtubule tip increases growth speeds up to four-fold and strongly reduces depolymerization events. Since tubulin concentration determines microtubule growth, our discovery shows a regulatory mechanism for enriching tubulin availability at strategically important locations: the growing microtubule tips.

Keywords: Microtubule, CLIP-170, EB3, microtubule dynamics, tubulin condensation, in vitro, liquid-liquid phase separation

\section{Introduction}

The microtubule cytoskeleton engages in a plethora of cellular processes, from organelle transport to cell division. To do so, the network dynamically modifies its structure in response to external cues and adapts its architecture to specific cellular functions. Microtubules themselves are highly dynamic polymers that can rapidly cycle between phases of polymerization and depolymerization, a characteristic which is critical for cytoskeletal re-organization (reviewed in Brouhard and Rice, 2018). In cells, microtubules polymerize at their plus-end by addition of GTP-tubulin. After GTPtubulin addition, GTP is gradually hydrolyzed, resulting in a GDP-tubulin shaft behind the tip. Once the stabilizing GTP-tubulin "cap" disappears from the plus-end, the microtubule switches from growing to shrinking, an event termed catastrophe (Howard and Hyman, 2009; Brouhard 2017, 2018). Conversely, microtubules can stop shrinking and switch to regrowth, an event termed rescue. The balance between growth and shrinkage is intimately linked to the addition of free tubulin to the growing microtubule (Walker et al., 1991; Voter et al., 1991); thus, regulating tubulin availability is critical to ensure the overall integrity of the microtubule network. 
In addition to these intrinsic modes of regulation, microtubule dynamics can be fine-tuned by Plus Tip-Interacting Proteins (+TIPs) (reviewed in Akhmanova and Steinmetz, 2010). +TIPs are functionally independent and structurally diverse microtubule regulators that concentrate at growing microtubule ends while exhibiting a weak affinity for the microtubule shaft. This unique localization results in a 0.5-2 $\mu \mathrm{m}$ long +TIP-network at the growing microtubule tip (Seetapun et al., 2012). Key integrators of these +TIP-networks are the End-Binding proteins (EBs), as they autonomously bind to GTP-tubulin at growing microtubule ends and recruit a battery of nonautonomously binding +TIPs (reviewed in Galjart, 2010). Within the EB family, higher eukaryotes express three proteins termed EB1, EB2, and EB3. These proteins increase microtubule plus-end dynamics by promoting catastrophes and increasing growth speed in vitro (Bieling et al., 2008; Vitre et al., 2008; Komorova et al., 2009; Gouveia et al., 2010).

A key accessory protein recruited to plus ends by the EBs is the Cytoplasmic Linker Protein of $170 \mathrm{kDa}$ (CLIP-170), which increases microtubule rescue frequency and growth speeds (Perez et al., 1999; Arnal et al., 2004; Bieling et al., 2007; Bieling et al., 2008; Komorova et al., 2002, 2005). CLIP-170 consists of a microtubule binding "head" domain in its N-terminus, followed by a central coiled-coil region and a zinc-knuckle domain, hereafter referred to as the "C-terminal region" (Pierre et al., 1992; Pierre et al., 1994; Diamantopolous et al., 1999). Most studies of CLIP-170 function in vitro have focused on truncated versions containing only the monomeric head domain (H1) or the head domain with a small extension that allows dimerization (H2); thus, it is unclear how full-length CLIP-170 contributes to microtubule dynamics. Early studies of CLIP-170 expression in cells revealed that in addition to its microtubule plus-end localization, it also formed cytoplasmic "patches" that co-localized with EB1 and the dynein-activating protein dynactin (Pierre et al., 1994; Goodson et al., 2003). Based on the physical properties of these patches, it has recently been suggested that they form by liquid-liquid phase separation (LLPS) (Wu et al., 2021).

LLPS is the process by which molecules spontaneously condensate into droplets and de-mix from their surrounding solution, resulting in the co-existence of two unique liquid phases (reviewed in Boeynaems et al., 2018; Hyman et al., 2014; Shin and Brangwynne, 2017). Recently, LLPS has been implicated in driving microtubule-related processes, including spindle assembly (Zhang et al., 2015), nucleation of acentrosomal and branched microtubules (Hernández-Vega et al., 2017; King and Petry, 2020), and centrosome maturation (Woodruff et al., 2017; Jiang et al., 2021). A key shared feature of these processes is the co-condensation of tubulin with LLPS-potent microtubule-associated proteins to catalyze biochemical reactions. Along these lines, condensation of a microtubule +TIP-network has been proposed based on transient multivalent interactions between the +TIPs (Akhmanova and Steinmetz, 2015; Wu et al., 2021). Despite this, LLPS at the microtubule plus-end has never been shown.

In this study, we use tandem in-cell and in vitro approaches to investigate +TIP condensation at the microtubule plus-end. We provide the first evidence of +TIP LLPS at nanomolar concentrations in the absence of crowding agents. The EB3 and CLIP-170-containing droplets co- 
condense tubulin, causing local enrichment of tubulin at the growing microtubule tip. We demonstrate that this mechanism of tubulin co-condensation drives microtubule growth, while the growth-promoting activity depends on the multivalency of the +TIP-network. In cells, the multivalent +TIP-network also shows liquid-like properties at microtubule ends. This local increase in tubulin concentration could be a mechanism to facilitate the fast polymerization rates observed in cells.

\section{Results}

\section{CLIP-170 forms biomolecular condensates in cells and in vitro}

Previous studies indicate that CLIP-170 overexpressed in cells has the potential to undergo LLPS (Pierre et al., 1994; Goodson et al., 2003; Wu et al., 2021). Indeed, overexpression of mCherryCLIP-170 in CRISPR/Cas9 knock-in GFP-tubulin RPE-1 cells resulted in cytosolic CLIP-170 patch formation in addition to microtubule plus-end tracking (Figure 1A). These patches displayed liquid properties, undergoing fusion and fission within $5 \mathrm{sec}$ (Figure 1B; Movies 1 and 2). Fluorescence recovery after photobleaching (FRAP) revealed that CLIP-170 diffuses highly within these patches and showed a high protein exchange rate with the pool outside the patch, with a recovery half-life and mobile fraction of 27.5 seconds and $93 \pm 32 \%$, respectively (Figure 1C and Movie 3). We conclude that these CLIP-170 containing patches are droplets.

To address whether CLIP-170 alone undergoes phase separation, we purified recombinant fulllength GFP-CLIP-170 (FL-CLIP) from insect cells and reconstituted its phase separation properties in vitro. In the absence of other proteins, FL-CLIP condensed into spheres at concentrations as low as $100 \mathrm{nM}$ (Figure 1D, left panel). To estimate the amount of FL-CLIP in the dense phase we used a high throughput confocal microscopy-based approach. Briefly, i) protein mixtures were incubated in 384-well plates, ii) proteins in the dense phase were centrifuged onto the bottom of the wells, iii) wells were imaged using an automated confocal microscope, and iv) surface coverage of protein in the dense phase on well-bottoms was measured (Figure S1A; see Methods for details). A hallmark property of LLPS-potent proteins is that they condensate in a concentration-dependent manner and in response to the presence of crowding agents (Alberti et al., 2018). In line with this, the sphere size and amount of FL-CLIP in the dense phase increased in a concentration dependent manner even in the absence of crowding agents (Figures $1 \mathrm{D}$ and $\mathrm{S} 1 \mathrm{~B})$. Addition of a crowding agent, $2 \%$ polyethylene glycol (PEG), increased the amount of FLCLIP in the dense phase by 1.5 -fold but reduced sphere size (Figures S1C and D).

In line with the case in cells, FL-CLIP spheres displayed liquid properties such as fusion, although 5-times slower than what we observed in cells (Figure 1E and Movie 4). We further interrogated the liquid properties of these spheres by FRAP, which confirmed that FL-CLIP diffuses within the spheres with a half-life of $27.5 \mathrm{~s}$, identical to the recovery speed in cells. However, FL-CLIP exchange dynamics were reduced three-fold with a mobile fraction of $28 \pm 12 \%$ (Figure $1 \mathrm{~F}$ and Movie 5). Collectively, these results show that FL-CLIP undergoes LLPS and forms droplets in 
vitro at nanomolar concentration and in the absence of further proteins or crowding agents. We hypothesize that the discrepancies between the material properties of the droplets in cells and in vitro are due to the presence of additional proteins and/or CLIP-170 post-translational modifications in the cell droplets.

The CLIP-170 C-terminal region drives CLIP-170 into the dense phase

We next investigated which domains of CLIP-170 drive droplet formation. We expressed in RPE1 cells two truncated forms of CLIP-170: H1, a monomeric N-terminal head domain; or H2, a dimeric form with an additional short coiled-coil extension (Figure 2A) (Pierre et al., 1992, 1994; Diamantopoulos et al., 1999; Goodson et al., 2003). In line with previous observations, we saw that $\mathrm{H} 1$ and $\mathrm{H} 2$ displayed microtubule plus-end tracking activity in cells, but did not form any cytosolic droplets (Figure 2B and Movies 6 and 7) (Pierre et al., 1994; Goodson et al., 2003). To understand to which extent the C-terminal region is necessary for CLIP-170 to undergo LLPS, we purified recombinant human $\mathrm{H} 1$ and $\mathrm{H} 2$ in bacteria and measured their ability to condensate in vitro at nanomolar concentrations. While FL-CLIP phase separated in the absence of other factors, H1 showed faint irregular-shaped aggregation but no droplet formation even in presence of $10 \%$ PEG (Figures 2C, S2B and S2C). In contrast to the case in cells, at nanomolar concentration H2 underwent condensation in vitro, although with a 300 -fold reduction of protein in the dense phase and a 25-fold reduction in droplet size compared to FL-CLIP (Figures 2C-E and S2A). Addition of $2 \%$ PEG increased $\mathrm{H} 2$ condensate formation by 3-fold but did not affect condensate size (Figures 2C-D, S2B and S2C). These results show that while the monomeric H1 is sufficient to track the growing microtubule tip, the dimeric form of CLIP-170 is necessary to undergo LLPS and that the $\mathrm{C}$-terminal region robustly drives CLIP-170 condensation.

\section{EB3 undergoes LLPS and co-condenses with CLIP-170}

CLIP-170 requires the presence of EBs to localize to growing microtubule ends (Dixit et al., 2009, Bieling et al., 2008). This prompted us to ask whether purified FL-CLIP and EB3 could cocondense in vitro. We first studied EB3 alone and observed that it has the capacity to undergo LLPS at micromolar concentrations: compared to $48 \pm 17 \mu \mathrm{m}^{2}$ large droplets observed for $400 \mathrm{nM}$ FL-CLIP, at $2.4 \mu \mathrm{M}$ EB3 phase separated into many small $1.9 \pm 0.6 \mu \mathrm{m}^{2}$ droplets, resulting in a 3.5-fold reduced surface coverage (Figures 3A and S3A). Both the droplet size and EB3 fraction in the dense phase were comparable between 0 and $2 \%$ PEG (Figure 3A).

Given their individual propensities for condensation, we hypothesized that a multivalent EB3/FLCLIP network may robustly drive phase separation even further. Indeed, EB3/FL-CLIP networks formed droplets that covered the surface 2.5-fold higher than FL-CLIP droplets alone (Figure 3B). Importantly, EB3/FL-CLIP networks had a synergistic effect on droplet formation, increasing the surface coverage by $40 \%$ when compared to the sum of the surface coverages of EB3 alone and FL-CLIP alone (Figure 3C). Repeating the EB3 experiment with $\mathrm{H} 2$ reduced surface coverage by 1.5-fold compared to the presence of FL-CLIP, and H1 further decreased surface coverage by 2.5fold (Figures 3D and S3B). These data demonstrate that CLIP's C-terminal region is essential for 
a highly multivalent EB3-CLIP network formation. We further show that EB3 and CLIP-170 can undergo LLPS both independently, and that when acting as an ensemble, the amount of proteins in the dense phase synergistically increases.

\section{CLIP-170 and EB3 condense tubulin in vitro}

A CLIP-170 dimer has as many as 8 tubulin binding sites, can bind tubulin in vitro, and colocalizes with tubulin in cells (Figure 4A) (Pierre et al., 1994 et al., 1999; Perez et al., 1999; Gupta et al., 2010). We therefore asked if CLIP-170 droplets can co-condense tubulin in vitro. Tubulin alone did not form droplets at micromolar concentrations; even in the presence of 5\% PEG only aggregation was observed (Figure S4A). However, when 200 nM FL-CLIP was mixed with 400 $\mathrm{nM}$ Atto561-tubulin, the two proteins phase separated into $26.8 \pm 12 \mu \mathrm{m}^{2}$ droplets (Figures $4 \mathrm{~B}$ and $\mathrm{S} 4 \mathrm{~B})$. We repeated these experiments with $\mathrm{H} 1$ and found irregular shaped aggregation with tubulin but no droplet formation (Figure 4B). We next asked whether EB3 could condense tubulin, based on its intrinsic phase separating capacity (Figure 3A). Indeed, EB3 also co-condensed tubulin, although into much smaller $1 \pm 0.07 \mu \mathrm{m}^{2}$ droplets (Figures 4C and S4C). Strikingly, FL-CLIPdroplets enriched tubulin 40-fold more than EB3 droplets, even at 6-fold lower FL-CLIP concentrations (Figure 4C). Based on the multivalency of a EB3/CLIP-170/tubulin network (Figure 4A), we then asked whether FL-CLIP and EB3 could cooperatively increase the amount of tubulin condensation compared to FL-CLIP alone. Interestingly, we observed that EB3/FLCLIP networks were 4-fold less efficient at condensing tubulin than pure FL-CLIP (Figure 4C). We hypothesize that addition of tubulin to EB3/FL-CLIP droplets partially compete for EB3/FLCLIP interactions and thereby reduces the valency of the network. Collectively, these results show that FL-CLIP can condense tubulin effectively and that EB3 reduces the tubulin condensation capacity of FL-CLIP droplets.

\section{Tubulin co-condenses with CLIP-170 in cells}

Overexpressing GFP-CLIP-170 in RPE-1 cells revealed that CLIP-170 droplets colocalized with areas of high tubulin fluorescent intensity (Figure 4D, left panel) (Perez et al., 1999). However, it was not possible to distinguish if these areas corresponded to microtubule bundles, or if the local increased signal resulted from tubulin condensation. To address this question, we depolymerized the microtubule network with $5 \mu \mathrm{M}$ nocodazole (Figure 4D, right panel). After microtubule depolymerization, tubulin showed robust co-localization with CLIP-170 droplets (Figure 4D). Within the CLIP-170 droplets tubulin fluorescence intensity was 2.4-fold higher compared to the cytoplasm (Figure 4D, graph). Importantly, even in WT cells small condensates of endogenous tubulin and CLIP-170 were found to co-localize after microtubule network depolymerization (Figure S4D). Contrary to our in vitro experiments, H2 and EB3 did not co-condense tubulin in cells (Figure S4E). After depolymerization of the microtubule network EB3 was cytoplasmic with few small condensates, but showed no co-condensation with tubulin (Figure S4E). We conclude that CLIP-170, but not EB3, can co-condense tubulin in cells. 
In cells, +TIP-networks show liquid-like properties

In cells EB3 and CLIP-170 are prominent members of the +TIP-network at the growing microtubule tip. Our in vitro results that low concentrations of EB3 and FL-CLIP undergo LLPS prompt the possibility that cellular +TIP-networks could be conducive to LLPS. To this end, we overexpressed GFP-CLIP-170 in live RPE-1 cells to analyze the characteristic profile of +TIPnetworks (Perez et al., 1999; Henrie et al., 2020). We frequently saw trailing remnants that split off from +TIP-networks in a process reminiscent of droplet fission, and the remnants dissolved over time (Figure 5C and Movie 8). Analysis of plus-end fluorescence intensity profiles in fixed cells revealed that $80 \%$ of the GFP-CLIP-170 networks left behind CLIP-170 remnants (Figures 5A and 5D). Overexpression of GFP-H2 and GFP-H1 resulted in similar +TIP-profiles, although with 5-fold less remnants (Figures 5A and 5D). These results show that the regions of CLIP-170 required for phase separation in vitro are also necessary for remnant formation in cells. Based on the characteristic appearance of remnants and that they dissolve over time, the results imply that CLIP-170 remnants form by fission from a liquid-like +TIP-network (Figure 5C and Movie 8).

To rule out that the observed remnant formation is not an artifact of overexpression, we measured plus-end fluorescence intensity profiles of endogenous CLIP-170. In line with overexpression studies, we observed that for endogenous CLIP-170, 70\% of the +TIP-networks had remnants (Figures 5B and 5D). Endogenous EB3 followed a comparable fluorescence profile, with $~ 75 \%$ of the networks having remnants, consistent with a recent study that observed EB3 remnants upon overexpression (Mustyatsa et al., 2019). Co-staining of EB3 and CLIP-170 revealed that many +TIP-networks displayed fluorescence profiles with distinct EB3 and CLIP-170 remnant colocalization (Figure 5E). To probe if the characteristic profile of +TIP-networks results from liquid properties of the network, we treated mCherry-EB3 expressing cells with 1,6-hexanediol, an aliphatic alcohol that inhibits LLPS by perturbing hydrophobic interactions (Kroschwald et al., 2017). While untreated cells exhibited $3 \mu \mathrm{m}$ long +TIP-networks, upon hexanediol treatment, the profile of the +TIP-network reduced to $\sim 1.5 \mu \mathrm{m}$ foci (Figure 5F, 5G and Movie 9). This indicates that network length is influenced by the liquid-like properties of the +TIP network. Together, these results imply that EB3 and CLIP-170 can form a liquid-like +TIP network in cells.

\section{EB3/CLIP-170 depletion reduces microtubule growth speeds in cells}

Our findings that +TIPs undergo LLPS and that a multivalent EB3/CLIP-170 network condenses tubulin raises the possibility that cells use EB3/CLIP-170/tubulin co-condensation to drive microtubule growth by enriching tubulin at the growing tip. To this end, we used siRNA to codeplete EB3 and CLIP-170 in GFP-tubulin RPE-1 cells and measured microtubule growth speeds (Figures S5A and S5B). The absence of either EB3 or CLIP-170 at the microtubule tip does not reduce growth speeds in cells (Komorova et al., 2002; Straube et al., 2007; Komorova et al., 2009). However, combined knockdown of EB3 and CLIP-170 reduced growth speeds by $20 \%$ (Figures S5 C and S5D). Measuring the impact of increased plus-end LLPS in cells by co-overexpression of EB3 and CLIP-170 proved difficult, because cells did not tolerate higher level co- 
overexpression (data not shown); we therefore reconstituted microtubule growth in presence of a "+TIP-droplet" (a +TIP-network capable of undergoing LLPS) in vitro.

+ TIPs wet microtubules and condense tubulin, which promotes microtubule growth

To reconstitute +TIP phase separation on microtubules, we polymerized microtubules in presence of $5 \mu \mathrm{M}$ tubulin, $1.8 \mu \mathrm{M}$ EB3 and $50 \mathrm{nM}$ FL-CLIP. Under these conditions, EB3/FL-CLIP coated the microtubule shaft and formed regular droplets along the shaft resembling the Rayleigh-Plateau instability (Figure 6A and Movie 10) (Plateau, 1873; Rayleigh, 1878, Setru et al., 2021). These droplets robustly co-condensed and enriched tubulin over time on the microtubule shaft (Figures $6 \mathrm{~A}$ and $6 \mathrm{~B}$ ). With this wetting of the microtubule by EB3/FL-CLIP/tubulin, we observed rapid microtubule growth speeds of $3.6 \mu \mathrm{m} / \mathrm{min}$ and very few catastrophe events or pauses in the growth phase (Figures 6B, 6C and S6C). When we repeated these experiments with less potent phase separating EB3/H2-networks, EB3/H2 coated the microtubule lattice and occasionally formed condensates (Figure 6B, left panel). However, growth speeds were 2-fold slower than for EB3/FLCLIP, catastrophe frequencies were increased 7-fold and pauses increased 30-fold (Figures 6B, 6C and S6C). In the absence of EB3, we did not observe microtubule binding or increased growth speeds for any CLIP constructs (Figures S6A and S6B). Collectively, these experiments demonstrate that EB3/FL-CLIP networks undergo LLPS on microtubules and can locally condense tubulin, which drives microtubule growth.

The transition from + TIP-networks to + TIP-droplets accelerates microtubule growth

To drive the proteins off the microtubule shaft and enrich them at the growing tip we increased the salt concentrations (for details see methods) (Telley et al., 2011; Bieling et al., 2014; Chen et al., 2021). As a first step, we reconstituted microtubule growth in presence of a +TIP-network, with either reduced (50 nM H2 $+1.8 \mu \mathrm{M} \mathrm{EB} 3$ ) or minimal (50 nM H1 + 1.8 $\mu \mathrm{M}$ EB3) LLPS activity (Figure 3D), implying reduced or no co-condensation of tubulin at the growing tip (Figure 4C). As observed previously, EB3 alone increased microtubule growth speeds by 1.5-fold and catastrophe frequency by 8-fold (Figures 6D and 6E) (Komorova et al., 2009; Gouveia et al., 2010). Addition of $\mathrm{H} 1$ or $\mathrm{H} 2$ to the assay with $\mathrm{EB} 3$ resulted in tip-tracking behavior by EB3/H1 and EB3/H2 networks, but did not further change any of the dynamic parameters compared to EB3 alone (Figure 6D, 6E and S6D). These results show that +TIP-networks with reduced or no capacity to condense tubulin have the same impact on microtubule dynamics as EB3 alone.

We next aimed to reconstitute a +TIP-droplet with the capacity to locally condense tubulin at the growing microtubule tip. Under the same buffer conditions as above, EB3/FL-CLIP tracked the microtubule and showed minimal shaft wetting. However, in presence of EB3/FL-CLIP microtubules grew at a speed of $4 \mu \mathrm{m} / \mathrm{min}$, a two-fold increase compared to analogous experiments with $\mathrm{EB} 3 / \mathrm{H} 2$ and $\mathrm{EB} 3 / \mathrm{H} 1$, and a four-fold increase compared to controls with tubulin alone (Figures 6D, 6E and Movie 11). Furthermore, catastrophe events were rarely observed under these conditions (Figures 6D and 6E), and when they occurred were rapidly followed by rescue events 
(Figure S6D). We then asked to what extent EB3/FL-CLIP +TIP-droplets accelerated tubulin netaddition to the growing microtubule ends compared to EB3/H2 +TIP-networks. To estimate the local increase of available tubulin for polymerization, we measured microtubule growth speeds in the presence of constant EB3/H2 concentrations while increasing tubulin concentrations. We found that a growth speed of $3.8 \mu \mathrm{m} / \mathrm{min}$ (the speed achieved by EB3/FL-CLIP in the presence of $5 \mu \mathrm{M}$ tubulin) corresponds to $14 \mu \mathrm{M}$ tubulin in presence of EB3/H2 (Figure $6 \mathrm{~F}$ and $6 \mathrm{G}$ ). This implies that EB3/FL-CLIP networks enrich tubulin at the microtubule tip at least 2.8-fold more efficiently than EB3/H2 networks. These results show that phase separation of EB3/FL-CLIP locally condenses tubulin at the growing tip, driving rapid and continuous microtubule growth.

\section{Discussion}

Rearrangements of the microtubule network architecture require spatiotemporal regulation of microtubule growth. A large body of work has highlighted the role of +TIPs that act as microtubule polymerases (such as XMAP215) or increase microtubule tip dynamics (such as the EBs) in promoting these highly regulated changes (Gard and Kirschner, 1987; Srayko et al., 2005; Brouhard et al., 2008; Bieling et al., 2008; Vitre et al., 2008; Zanic et al., 2013; Yang et al., 2016). Here, we establish a new mechanism by which +TIPs regulate microtubule growth: through the local condensation of tubulin at the microtubule tip. Our model of +TIP-driven tubulin condensation is based on the following observations: i) the +TIPs EB3 and CLIP-170 have the capacity to undergo LLPS in vitro and in cells (Figures 1-4), ii) phase separated EB3/FL-CLIP droplets can condense tubulin (Figure 4), iii) microtubule growth speed is increased and catastrophe frequency reduced when EB3/FL-CLIP undergo phase separation on microtubules (Figure 6B-F), and iv) a +TIP-droplet has the capacity to enrich tubulin nearly three-fold compared to the surrounding buffer (Figure 6G). As tubulin is the most important component for microtubule growth, we hypothesize that this tubulin enrichment is a mechanism to increase local tubulin availability at microtubule ends and ensure efficient polymerization uncoupled form cytoplasmic tubulin concentrations.

Despite enrichment of tubulin into CLIP-droplets, we were unable to robustly detect an increase in tubulin fluorescence at growing microtubule ends (Figure 6C and E, FL-CLIP + EB3 kymographs). At the experimental concentration of $5 \mu \mathrm{M}$ tubulin, we have 18 tubulin molecules within one 200x200x150 nm pixel under our TIRF conditions. Our estimated local condensation of $14 \mu \mathrm{M}$ tubulin in a +TIP-droplet (estimated cylinder of $50 \mathrm{~nm}$ diameter and $200 \mathrm{~nm}$ height) would correspond to an additional 3 tubulin molecules per pixel, while a $200 \mathrm{~nm}$ long microtubule fragment with 13 protofilaments grown at $5 \mu \mathrm{M}$ tubulin has $343(325+18)$ tubulin molecules per pixel. The local increase of 3 tubulin molecules per pixel at the dynamically growing tip is shaded by the dynamic addition and dissociation of tubulin at the growing tip. Therefore, with the current light microscopy resolution and sensitivity this increase of tubulin at the microtubule tip is hardly detectable. 
Phase separation of a +TIP-droplet is initiated at the growing microtubule tip. Our results here demonstrate, in addition to the function of microtubule growth regulation, a mechanism for precise control of spatiotemporal initiation and dissolving of a LLPS process. We hypothesize that the mechanism of LLPS initiation depends on conformational properties of the growing microtubule tip (GTP-cap), which leads to differential binding of EB3 to the tip over the shaft and subsequent recruitment of CLIP-170 to form a multivalent network. Then, upon reaching a critical CLIP-170 concentration, this network undergoes LLPS and co-condenses tubulin to drive microtubule growth. Once the GTP-cap is hydrolyzed, the +TIP-droplet dissolves. Whether the stoichiometry between EB3 and CLIP-170 has an impact on the LLPS process, as well as the distinct fluid properties and dynamics of an EB3/CLIP-170 droplet, merit investigation in future work.

Is phase separation a common feature of +TIPs? Studies performed in parallel to our work show that this phenomenon is conserved across evolution: +TIPs in budding yeast, fission yeast, and higher eukaryotes have recently been demonstrated to undergo phase separation (Maan et al., 2021; Meier et al., 2021; Song et al., 2021). Intriguingly, in line with our results, the yeast studies confirmed that the CLIP-170 homolog played a key role in the phase separation process, whereas LLPS potency of EB homologs varied between organisms. The role of different mammalian EB family members in regulating LLPS will be an interesting direction for future studies. The ability of these +TIP-networks to phase separate depends on intrinsically disordered regions (Maan et al., 2021; Song et al., 2021) and multivalent interaction modules (Figure 2; Meier et al., 2021), consistent with the observation that these features are highly evolutionarily conserved across +TIPs (Wu et al., 2021). Further studies will be necessary to investigate whether additional +TIPs contribute to the formation and regulation of +TIP-droplets.

Our work here and recent studies demonstrate that +TIP networks can behave like liquid condensates (Wu et al., 2021; Maan et al., 2021; Meier et al., 2021; Song et al., 2021). This work adds to the growing list of microtubule-related processes that are driven by LLPS and provides an exciting new paradigm for how cells can spatiotemporally control microtubule dynamics through local tubulin concentration (Zhang et al., 2015; Woodruff et al., 2017; Hernández-Vega et al., 2017; King and Petry, 2020; Jiang et al., 2021; Maan et al., 2021; Meier et al., 2021; Song et al., 2021). Interrogating the mechanical properties and composition of +TIP-droplets, as well as studying their regulation throughout the cell cycle, will be exciting avenues for future research.

\section{Acknowledgements}

We thank Thomas Surrey (Centre for Genomic Regulation, Barcelona, Spain) for providing the FL-CLIP-170 expression vector for insect cells, and Michel Steinmetz (Paul Scherrer Institut, Villigen PSI, Switzerland) for providing vectors for EB3 purification. We would also like to thank Peter Bieling (Max Planck Institute of Molecular Physiology, Dortmund, Germany) for helpful discussions regarding expression and purification of FL-CLIP-170, and Maria Hondele (University of Basel, Switzerland) for helpful discussions for phase separation experiments. We would like to thank Dimitri Moreau and Stefania Vossio from the ACCESS Geneva high-content 
microscopy facility for help with microscopy and data analysis. We also thank Oscar Vadas and Rémy Visentin of the Protein Platform (Faculty of Medicine, University of Geneva, Switzerland) for support in FL-CLIP-170 expression. We would also like to thank Karsten Kruse and Marcos Gaitan-Gonzales and for critically reading the manuscript.

JM has been supported by the SNSF, 31003A_182473; RTW has been supported by the NCCR Chemical Biology program; CA has been supported by the DIP of the Canton of Geneva, SNSF (31003A_182473), and the NCCR Chemical Biology program.

Author Contributions: JM and RTW performed and designed the experiments with the help of CA. MCV purified the proteins. JM, RTW, and CA analyzed data. RTW and CA wrote the manuscript.

Declaration of Interests: The authors declare no competing interests. 


\section{Figures 1-6}

A

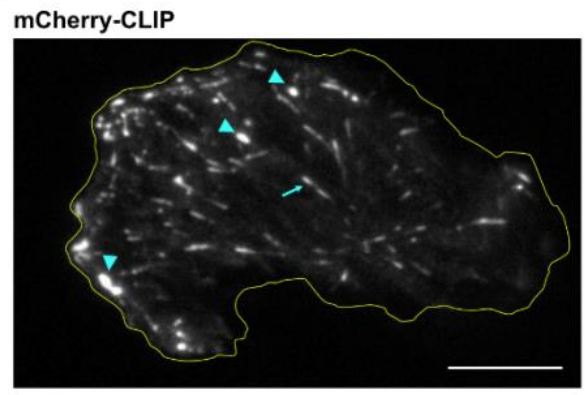

B

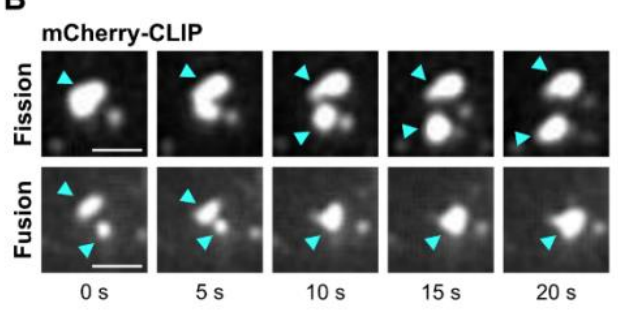

C
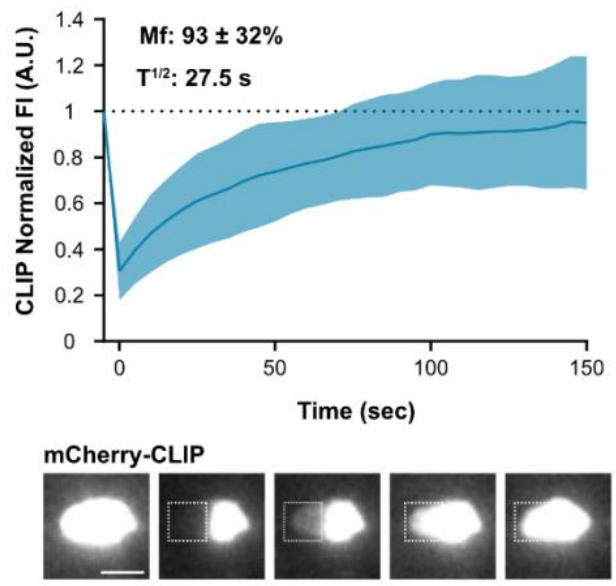

$-5 s$

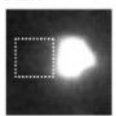

$0 \mathrm{~s}$

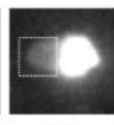

$5 \mathrm{~s}$

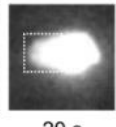

$20 \mathrm{~s}$

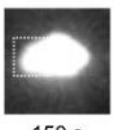

$150 \mathrm{~s}$
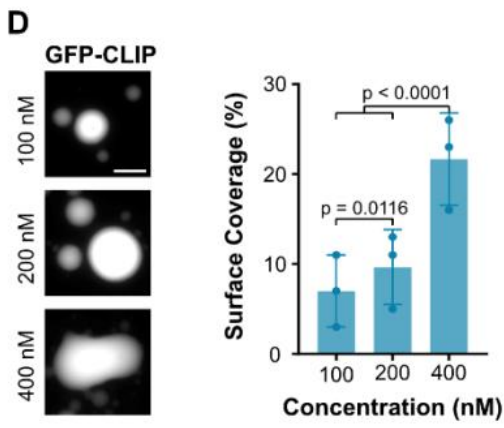

E

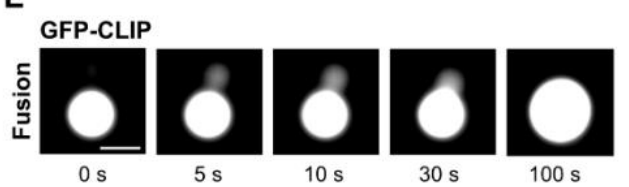

$\mathbf{F}$

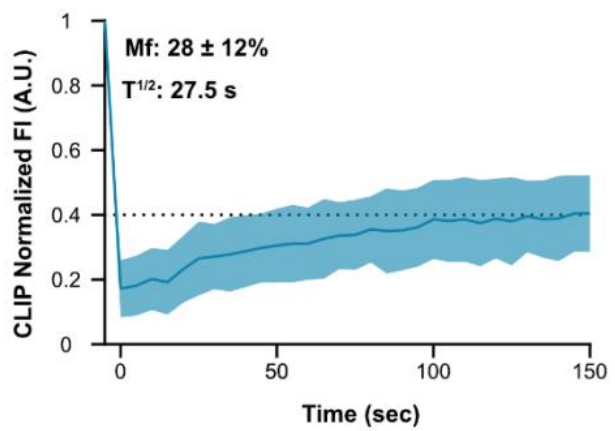

GFP-CLIP
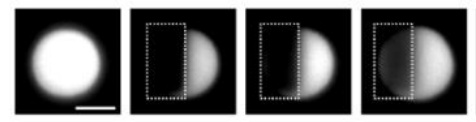

$100 \mathrm{~s}$

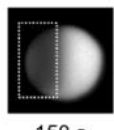

$150 \mathrm{~s}$

Figure 1: CLIP-170 condenses into droplets in cells and in vitro.

(A) Representative TIRF image of a RPE-1 cell transfected with mCherry-CLIP-170, showing the formation of cytoplasmic patches (cyan arrowheads) and +TIP-networks (cyan arrows). Scale bar: $10 \mu \mathrm{m}$. (B) Representative TIRF timelapse images of mCherry-CLIP-170 droplets undergoing fission (top panel, cyan arrowheads), and fusion (bottom panel, cyan arrowheads) in cells. Scale bar: $2 \mu \mathrm{m}$. (C) Representative TIRF images and recovery curve of mCherry-CLIP-170 patches after photobleaching (dashed box). Curve shows mean with SD of 5 individual experiments with a total of 38 droplets from 23 cells. Scale bar: $2 \mu \mathrm{m}$. D) Representative confocal images of purified GFP-FL-CLIP at indicated concentrations and quantification of the coverslip surface coverage by FL-CLIP. Statistics: two-tailed Student's $t$-test. Mean with SD from 3 independent experiments with a total of 27 fields of view. Scale bar: $20 \mu \mathrm{m}$. (E) Timelapse images of purified GFP-FLCLIP $(1 \mu \mathrm{M})$ undergoing fusion. Representative of 3 experimental replicates. Scale bar: $10 \mu \mathrm{m}$. 
(F) Representative images and recovery curve of purified GFP-FL-CLIP $(2 \mu \mathrm{M})$ droplets after photobleaching (dashed box). Curve shows mean with SD of 3 individual experiments with a total of 47 condensates. Scale bar: $5 \mu \mathrm{m}$.

A

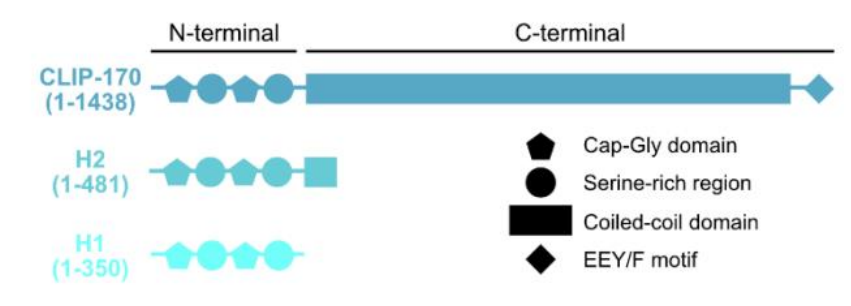

B
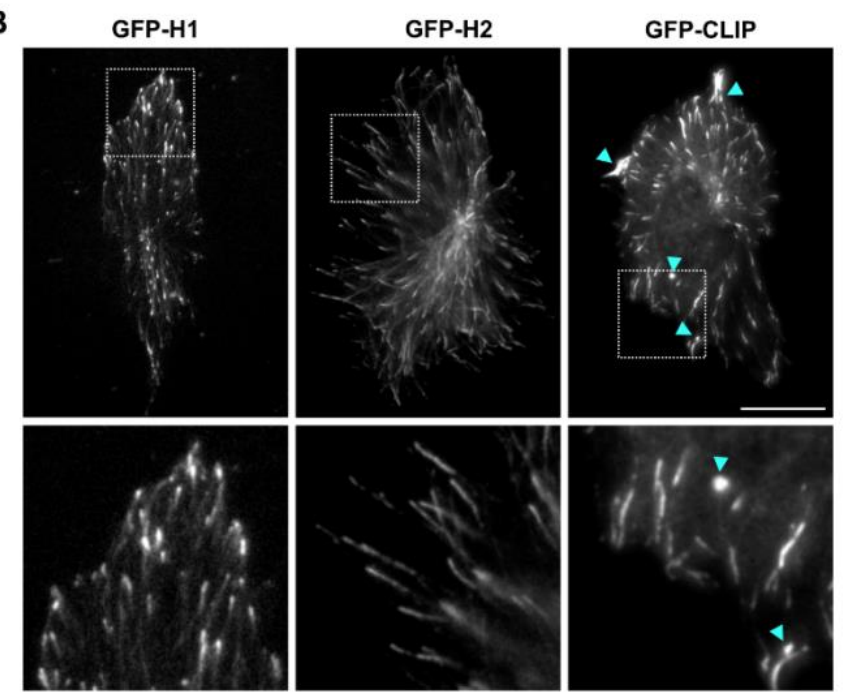
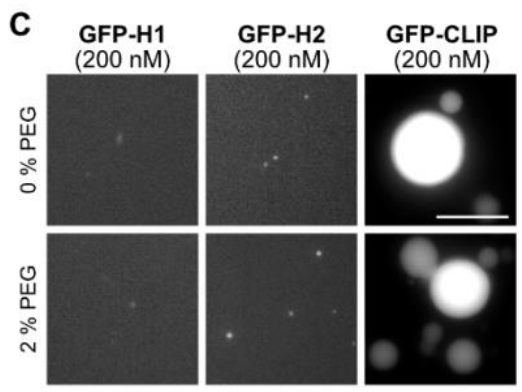

$\circ$

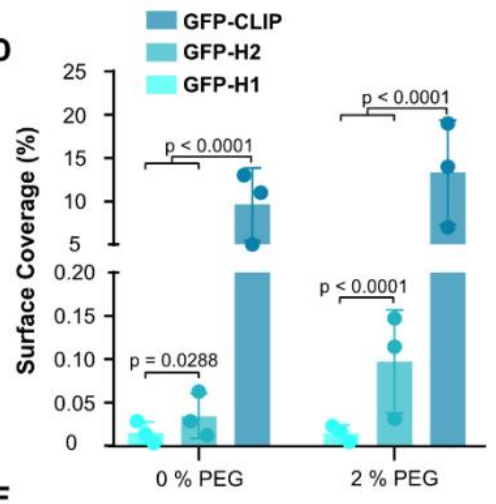

E

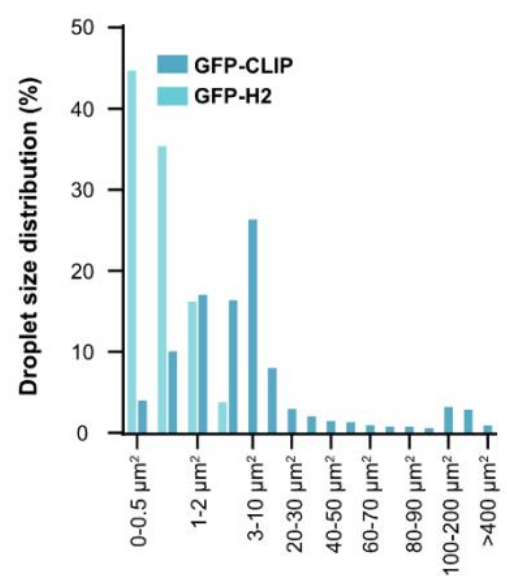

Figure 2: The C-terminal region drives CLIP-170 into the dense phase.

(A) Secondary structure of CLIP-170 (1-1438), H2 (1-481) and H1 (1-350) based on (Pierre et al., 1994; Diamantopolous et al., 1999; Goodson et al., 2003). Cap-Gly domains, Serine-rich domains, coiled-coil domain, and Cap-Gly-recognition motif (EEY/F) are denoted. (B) Representative images of fixed RPE-1 cells transfected with full length GFP-CLIP-170, GFP-H2 or GFP-H1 (top panel) with insets (bottom panel). Cyan arrowheads denote droplet formation in GFP-CLIPexpressing cell. Scale bar: $20 \mu \mathrm{m}$. (C) Representative confocal images of purified GFP-FL-CLIP, GFP-H2 and GFP-H1 each at $200 \mathrm{nM}$ in the absence (top panel) or presence (bottom panel) of $2 \%$ 
PEG. Scale bar: $20 \mu \mathrm{m}$. (D) Condensate surface coverage of the three constructs at indicated PEG concentrations. Mean with SD from 3 independent experiments with a total of 27 fields of view. Statistics: two-tailed Student's $t$-test. (E) Size distribution of GFP-FL-CLIP (200 nM) and GFP$\mathrm{H} 2(200 \mathrm{nM})$ droplets in the absence of PEG. Graph shows average size distribution from 3 independent experiments with a total of 27 fields of view.

A
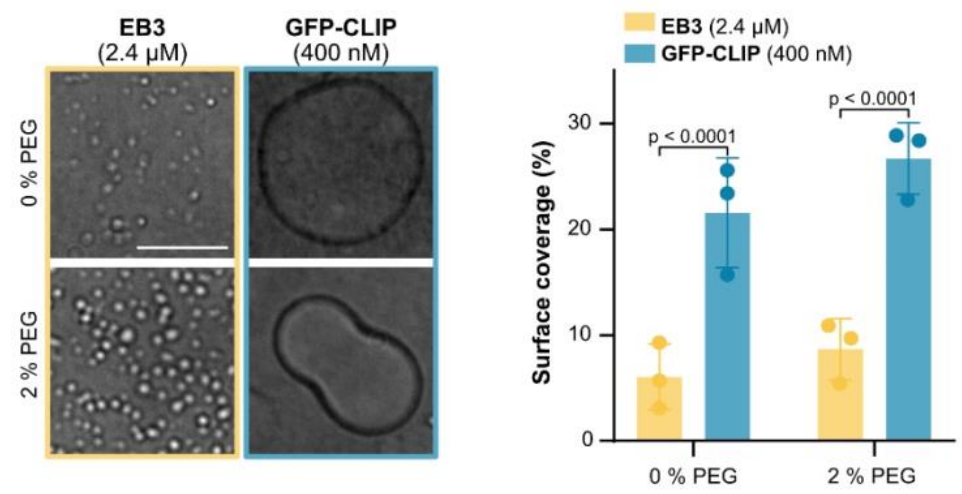

B
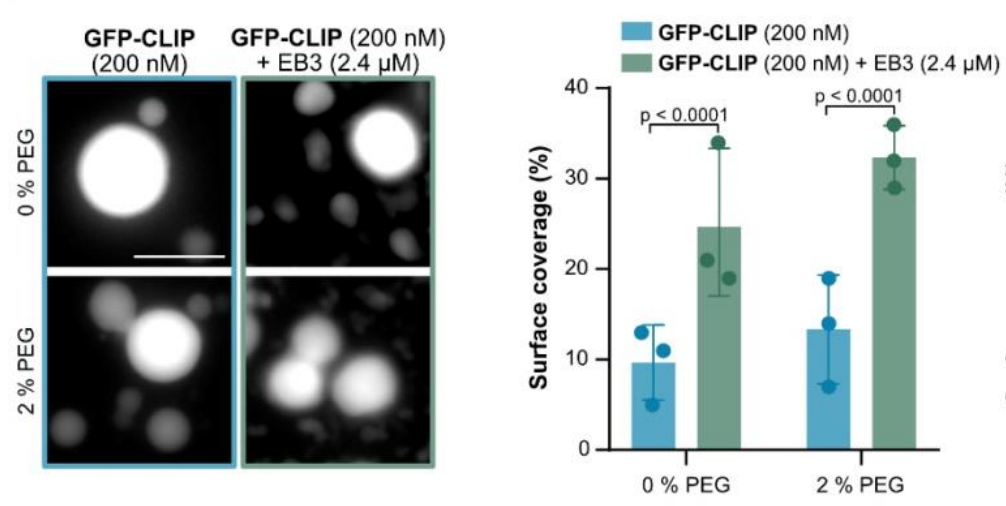

D
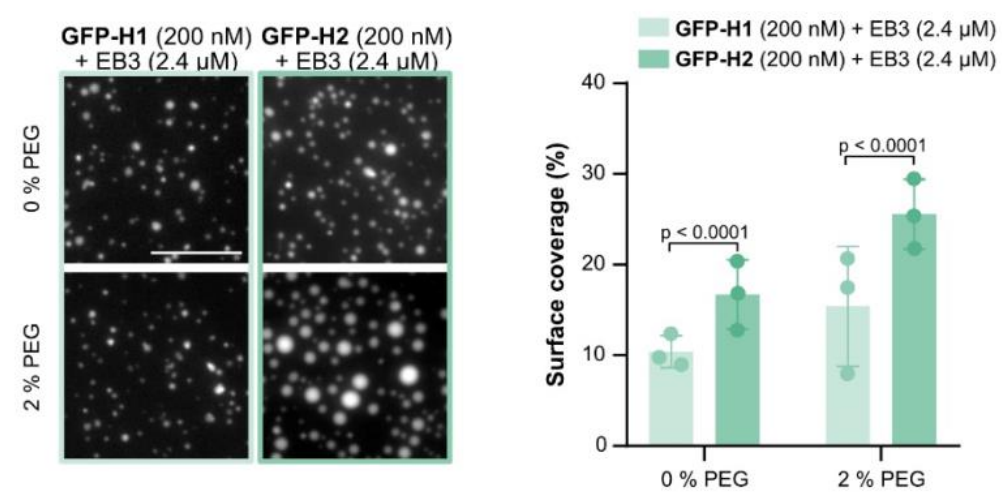

C

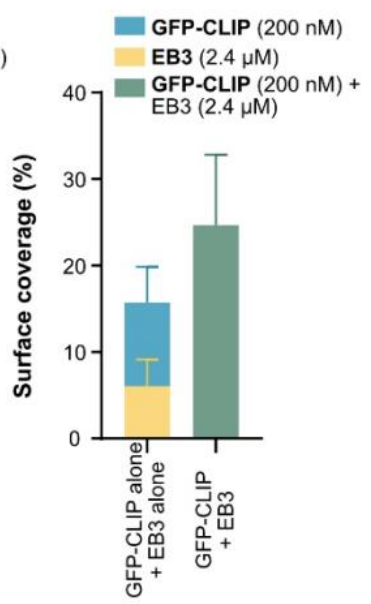

Figure 3: EB3 undergoes LLPS and co-condenses with CLIP-170 in vitro.

(A) Left: representative DIC images and quantification of purified EB3 $(2.4 \mu \mathrm{M})$ and GFP-FLCLIP (400 nM) in absence (top panel) or presence (bottom panel) of $2 \%$ PEG. Scale bar: $20 \mu \mathrm{m}$. 
Right: coverslip surface coverage of indicated proteins at denoted PEG concentrations. Mean with SD from 3 independent experiments with a total of 27 fields of view. Statistics: two-tailed Student's $t$-test. (B) Left: representative fluorescence confocal images and quantification of purified GFP-FL-CLIP in the absence (left) or presence (right) of EB3, and in the absence (top panel) or presence (bottom panel) of $2 \%$ PEG. Scale bar: $20 \mu \mathrm{m}$. Right: coverslip surface coverage of GFP-FL-CLIP (200 nM) in the dense phase with or without EB3 $(2.4 \mu \mathrm{M})$ in the presence of the indicated PEG concentrations. Mean with SD of from 27 fields of view from 3 independent experiments. Statistics: two-tailed Student's $t$-test. (C) Quantification of droplet surface coverage of EB3 and GFP-FL-CLIP alone compared to surface coverage of EB3/GFP-FL-CLIP droplet formation when undergoing synergistic LLPS in the absence of PEG. (D) Left: representative fluorescence confocal images and quantification of purified GFP-H1 (left) or GFP-H2 (right) in the presence of EB3, and in the absence (top panel) or presence (bottom panel) of $2 \%$ PEG. Scale bar: $20 \mu \mathrm{m}$. Right: condensate surface coverage of indicated GFP-H1 (200 nM) or GFP-H2 (200 $\mathrm{nM})$ in the presence of EB3 $(2.4 \mu \mathrm{M})$, and in the presence of the indicated PEG concentrations. Mean with SD from 3 independent experiments with a total of 27 fields of view. Statistics: twotailed Student's $t$-test. 
bioRxiv preprint doi: https://doi.org/10.1101/2021.09.13.459419; this version posted November 23, 2021. The copyright holder for this preprint (which was not certified by peer review) is the author/funder, who has granted bioRxiv a license to display the preprint in perpetuity. It is made available under aCC-BY-NC-ND 4.0 International license.

A

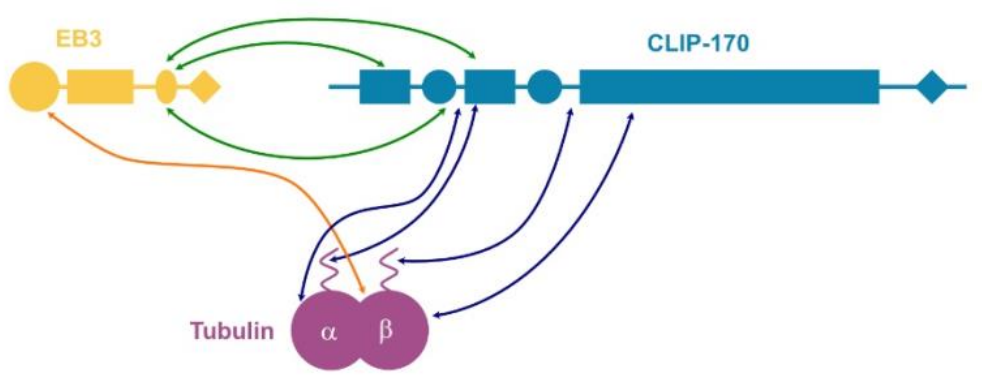

B
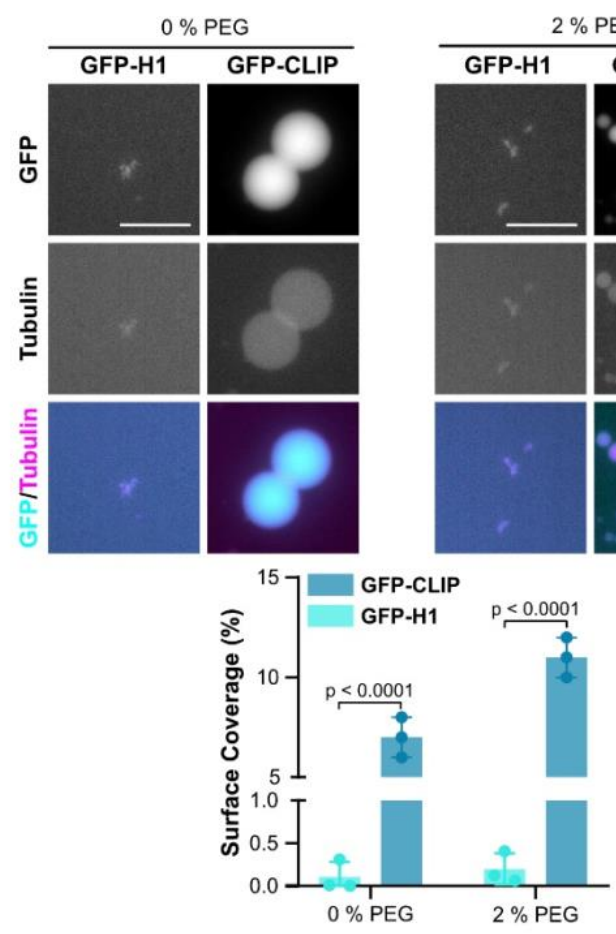

C
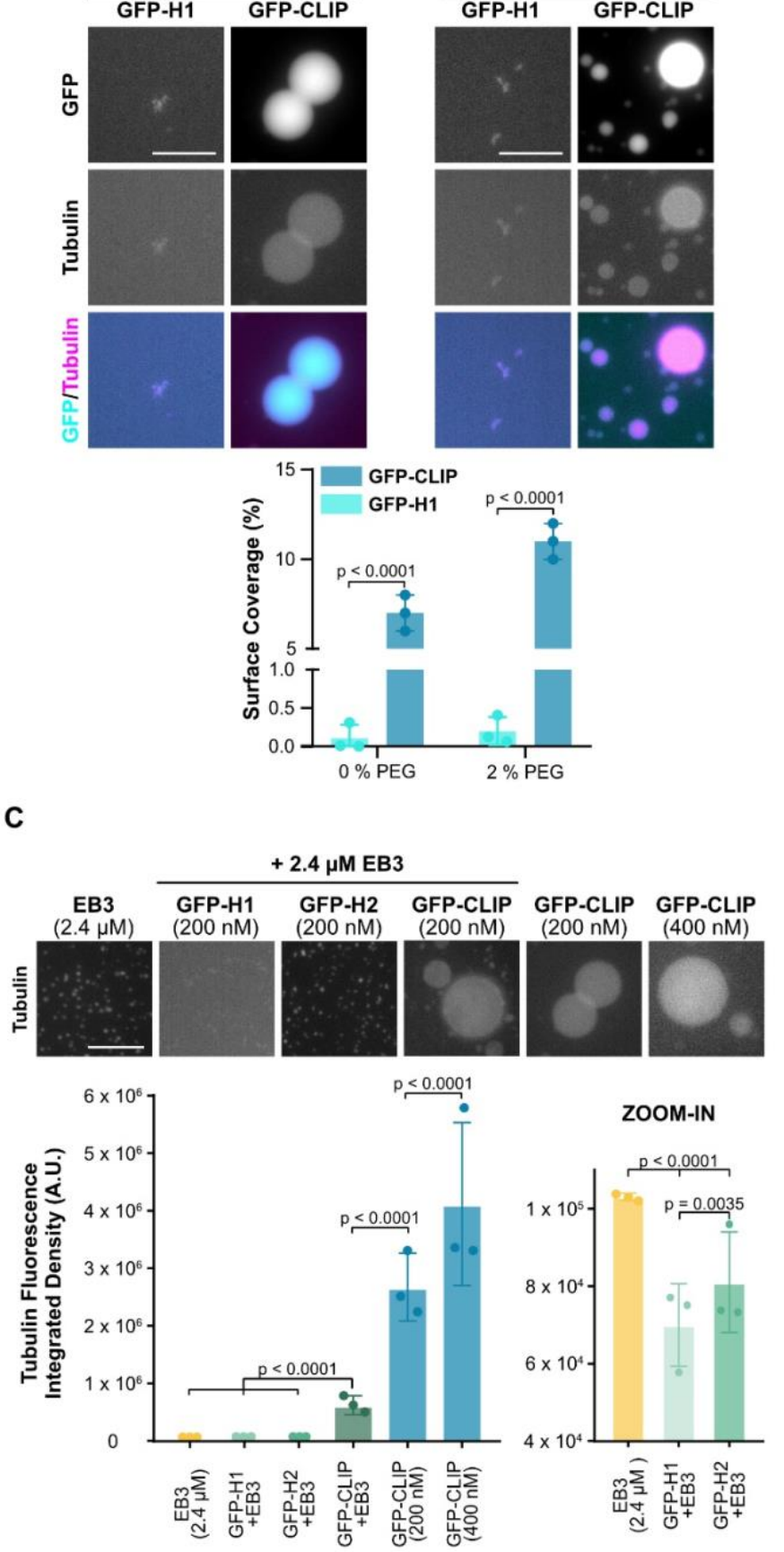

D

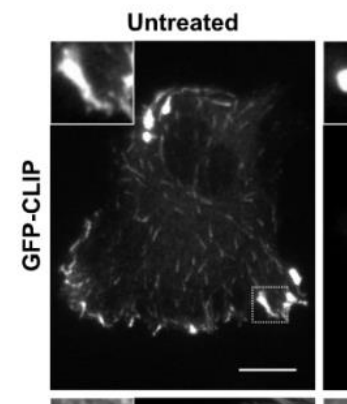

Nocodazole $(5 \mu \mathrm{M})$
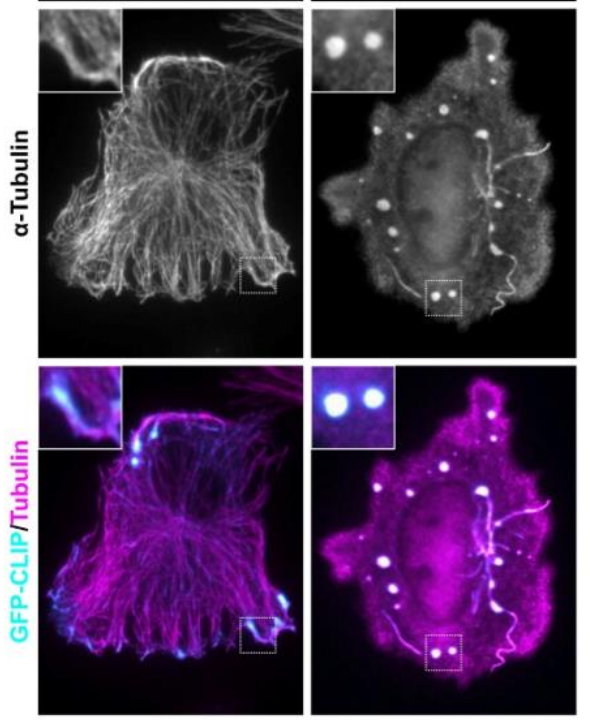

E

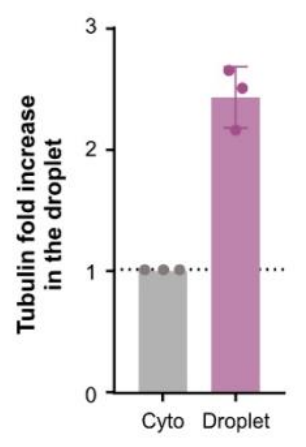


Figure 4: CLIP-170 and EB3 droplets condense tubulin.

(A) Cartoon schematic of domain interactions between EB3, CLIP-170, and tubulin based primarily on (Gupta et al., 2010; Chen et al., 2009; Bjelic et al., 2012). Interaction sites between EB3 and CLIP-170, EB3 and tubulin, and CLIP-170 and tubulin are shown with green, orange, and blue arrows, respectively. For simplification, monomers of EB3 and CLIP-170 are shown. (B) Top: representative confocal images of purified GFP-FL-CLIP and GFP-H1 each at $200 \mathrm{nM}$ with Atto-565-tubulin (400 nM); in the absence (left panel) or presence (right panel) of $2 \%$ PEG. Scale bar: $20 \mu \mathrm{m}$. Bottom: quantification of the coverslip surface coverage of tubulin in presence of GFP-FL-CLIP or GFP-H1. Mean with SD from 3 independent experiments with a total of 27 fields of view. Statistics: two-tailed Student's $t$-test. (C) Top: representative confocal images of Atto565-tubulin (400 $\mathrm{nM})$ in the presence of purified EB3 $(2.4 \mu \mathrm{M})$, GFP-H1 (200 nM) and EB3 (2.4 $\mu \mathrm{M})$, GFP-H2 $(200 \mathrm{nM})$ and EB3 $(2.4 \mu \mathrm{M})$, GFP-CLIP $(200 \mathrm{nM})$ and EB3 $(2.4 \mu \mathrm{M})$, and GFPCLIP (200 nM and 400nM) alone. Scale bar: $20 \mu \mathrm{m}$. Bottom: quantification of the integrated density of tubulin fluorescence under denoted conditions with zoom in for the first three conditions. Mean with SD from 3 independent experiments with a total of 27 fields of view. Statistics: two-tailed Student's $t$-test. (D) Representative images of fixed RPE-1 cells transfected with full length GFP-FL-CLIP and untreated (left panel) or treated with $5 \mu \mathrm{M}$ nocodazole for 1 hour (right panel) and stained for tubulin. Scale bar: $10 \mu \mathrm{m}$. Images are representative of 3 independent experiments. (E) Graph showing normalized tubulin fluorescence intensity in CLIPdroplets compared to cytoplasm in full-length GFP-CLIP-170 transfected RPE-1 cells treated with nocodazole. Graph shows mean with SD from 3 independent experiments with a total of 126 condensates from 26 cells. 
bioRxiv preprint doi: https://doi.org/10.1101/2021.09.13.459419; this version posted November 23, 2021. The copyright holder for this preprint
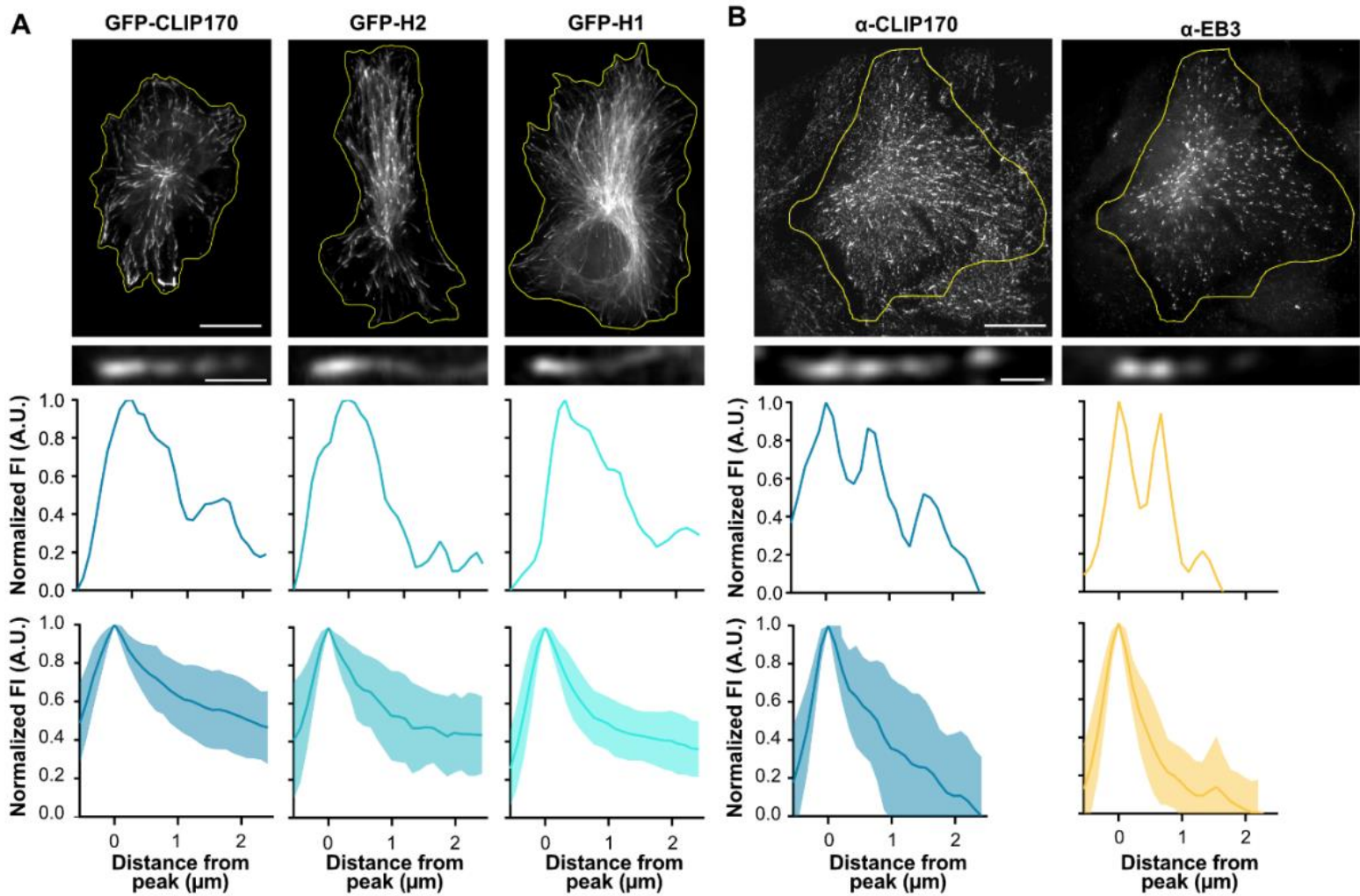

C
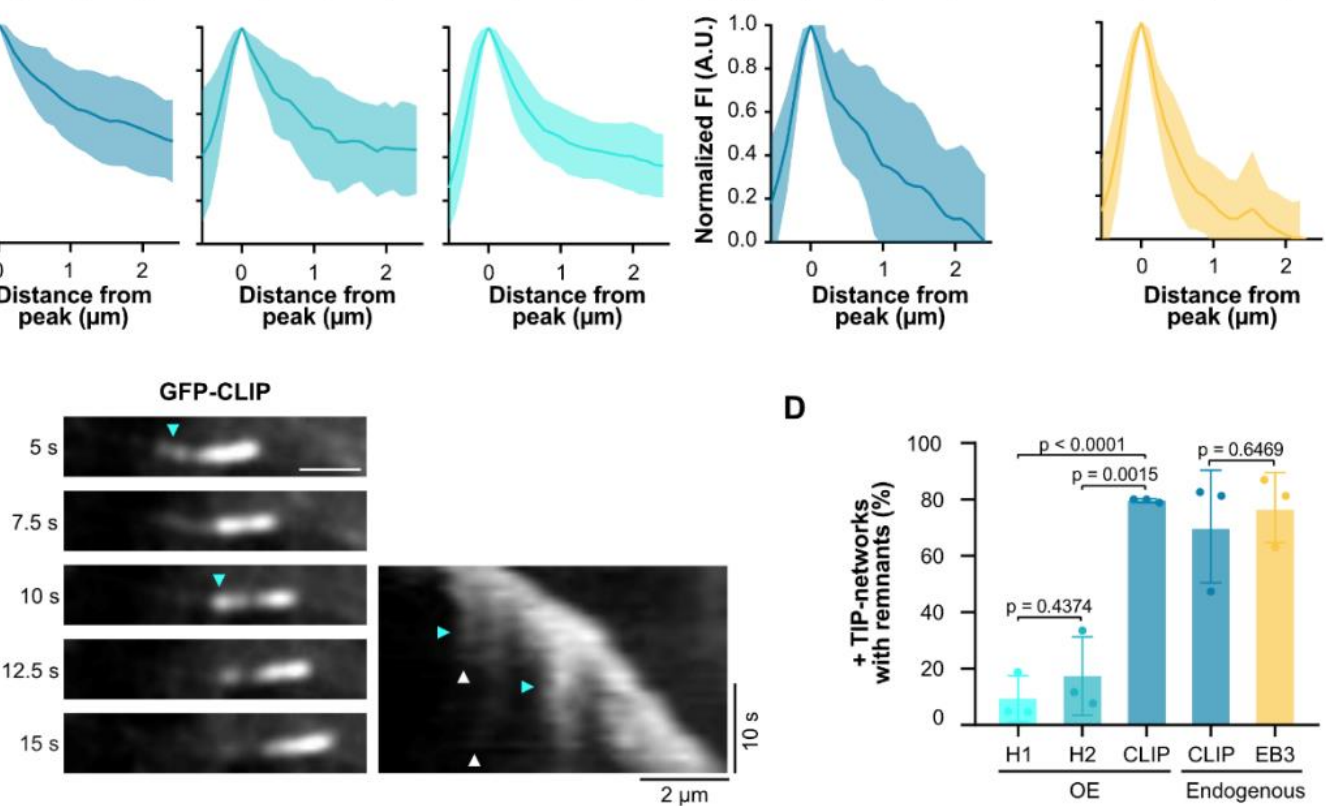

D
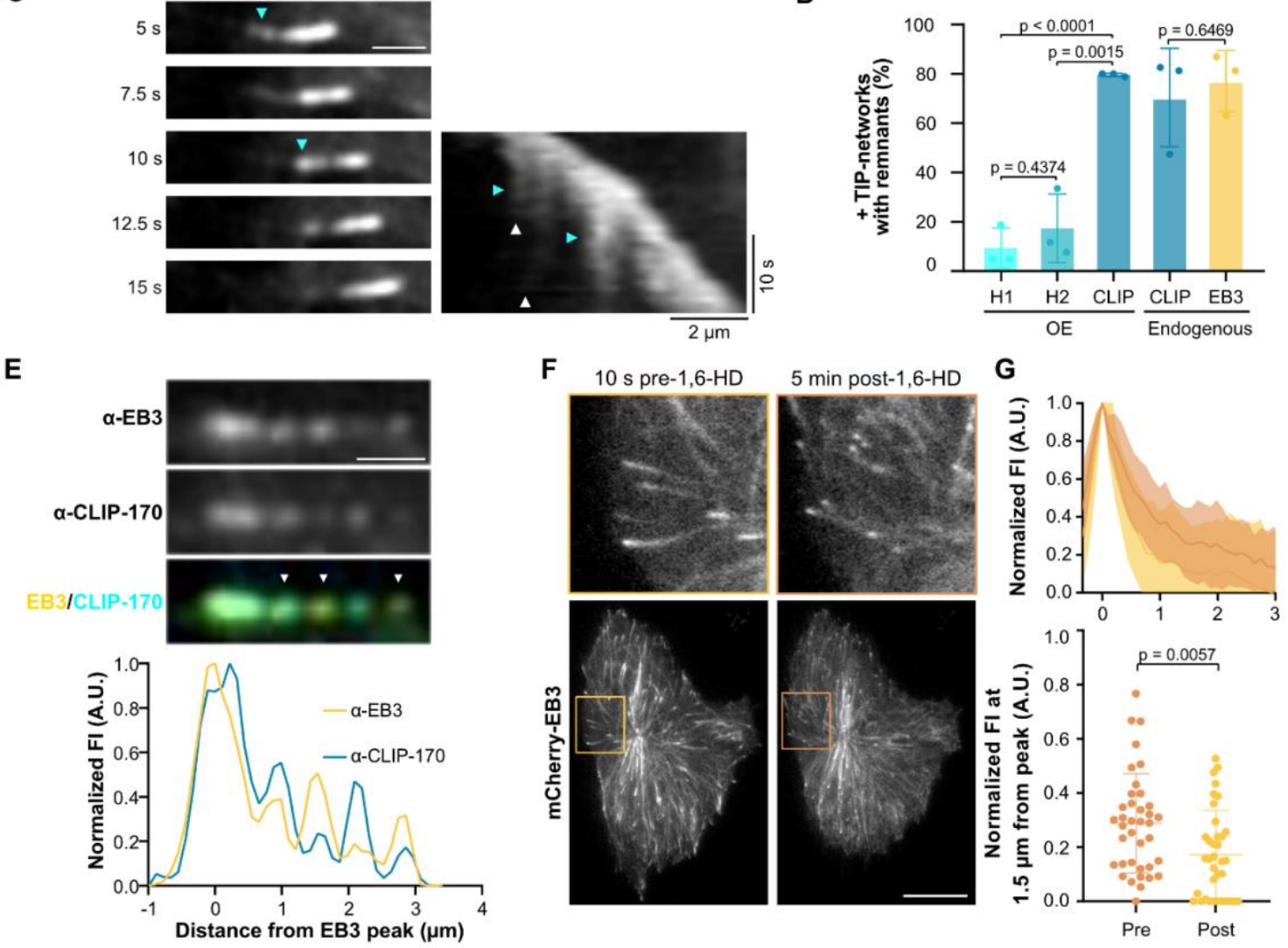
Figure 5: In cells the EB3/CLIP-170 + TIP-network displays liquid properties at microtubule tips. (A) Representative images (top) of fixed RPE-1 cells transfected with full length GFP-CLIP170, GFP-H2 and GFP-H1. Representative profiles of +TIP-networks with matching fluorescence linescans (bottom). Scale bars: $20 \mu \mathrm{m}$ whole-cell, $2 \mu \mathrm{m}$ insets. Below are quantified mean linescan profiles (dark line) with standard deviation (shaded area) from 3 independent experiments with a total of: H1 - 47 +TIP-networks from 22 cells; H2 - 33 +TIP-networks from 18 cells; CLIP-170 57 +TIP-networks from 22 cells. (B) Representative images (top) of fixed RPE-1 cells stained with antibodies to endogenous CLIP-170 and EB3. Representative profiles of +TIP-networks with matching fluorescence linescans (bottom). Scale bars: $20 \mu \mathrm{m}$ whole-cell, $2 \mu \mathrm{m}$ insets. Below are quantified mean linescan profiles (dark line) with standard deviation (shaded area) from 3 independent experiments with a total of 58 +TIP-networks from 12 cells for each condition. (C) Representative timelapse images (left) and kymograph (right) of +TIP-network from GFP-FLCLIP expressing RPE-1 cell. Cyan and white arrowheads denote remnants formation and dissolving respectively in both timelapse images and kymograph. Scale bar $(2 \mu \mathrm{m})$ (D) Percentage of +TIP-networks with remnants in fixed cells expressing the indicated CLIP constructs or stained with antibodies to endogenous CLIP-170 or EB3. Graph shows mean with SD from 3 independent experiments (cell/+TIP-network n-values denoted in legend from A and B). Statistics: one-way ANOVA test. (E) Representative +TIP-network from cells stained for endogenous EB3 and CLIP170 showing partial co-localization of EB3 and CLIP-170 remnants (white arrow heads) with corresponding fluorescence linescan below. Scale bar: $1 \mu \mathrm{m}$. (F): Representative images of GFPTubulin RPE-1 cells expressing mCherry-EB3 before and after 5-minute treatment with $5 \%$ 1,6hexanediol, with insets. (G) Top: mean fluorescence intensity profile of +TIP-networks before and after 1,6-hexanediol treatment. Bottom: normalized fluorescence intensity of +TIP-networks 1.54 $\mu \mathrm{m}$ away from the peak. Mean with SD of 36-38 +TIP-networks from 6 cells from 2 independent experiments. Statistics: paired t-test. Scale bars: $20 \mu \mathrm{m}$. 
bioRxiv preprint doi: https://doi.org/10.1101/2021.09.13.459419; this version posted November 23, 2021. The copyright holder for this preprint (which was not certified by peer review) is the author/funder, who has granted bioRxiv a license to display the preprint in perpetuity. It is made available under aCC-BY-NC-ND 4.0 International license.

A
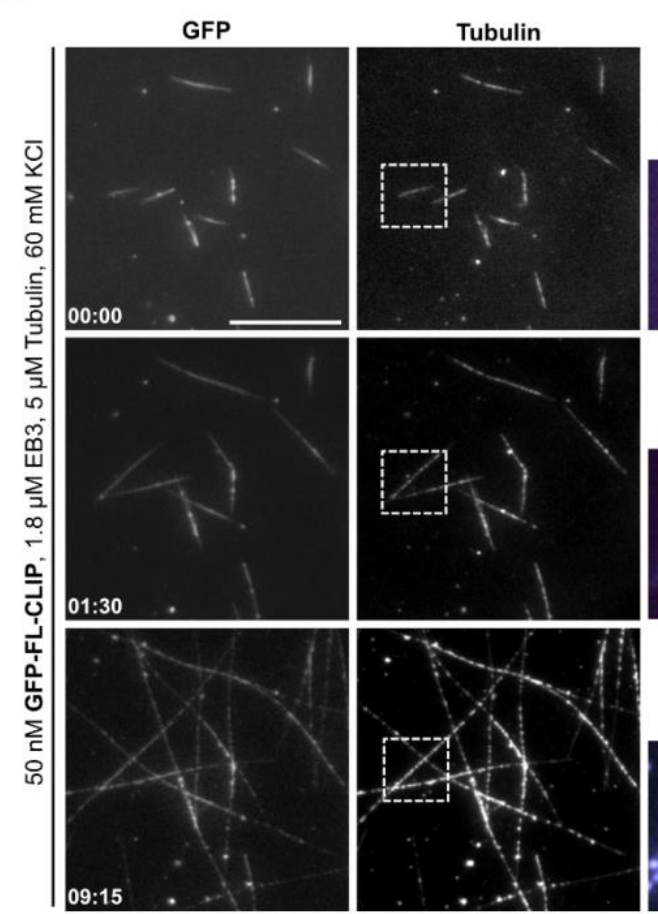

D

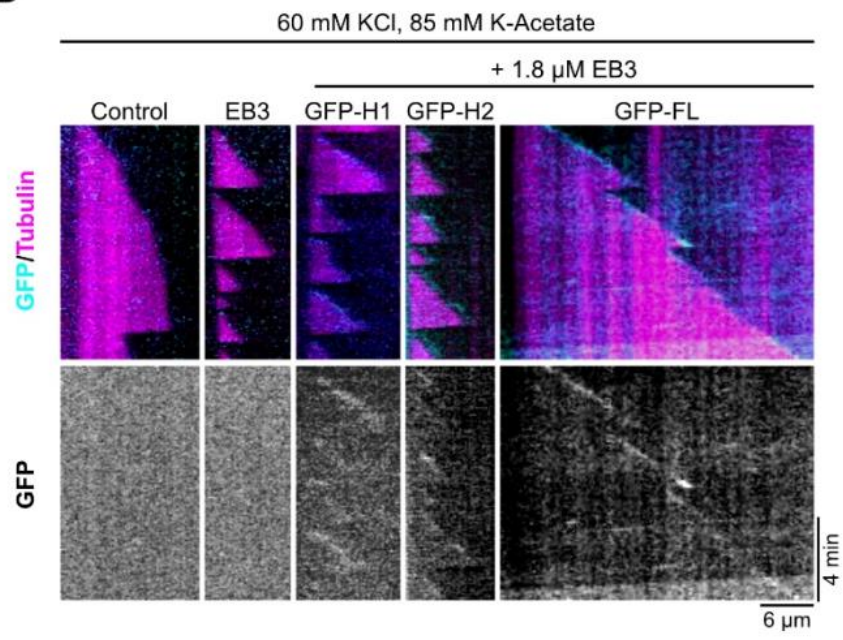

F

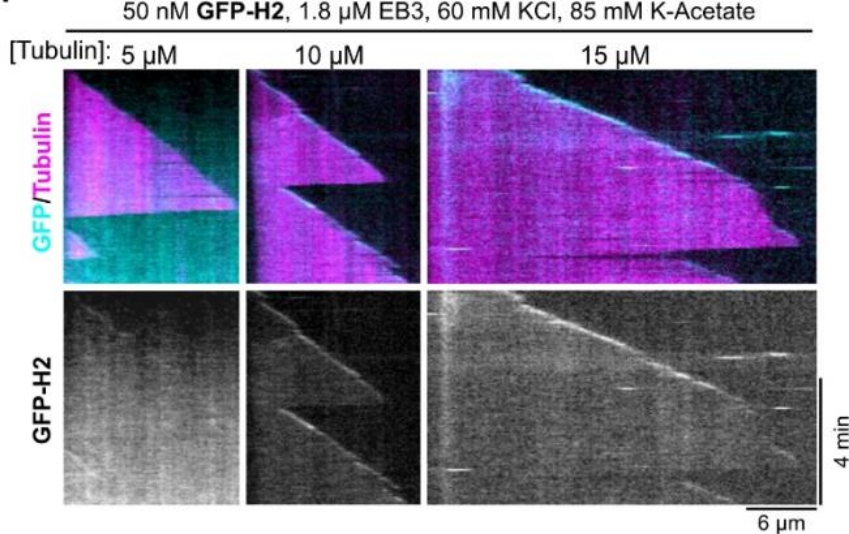

B

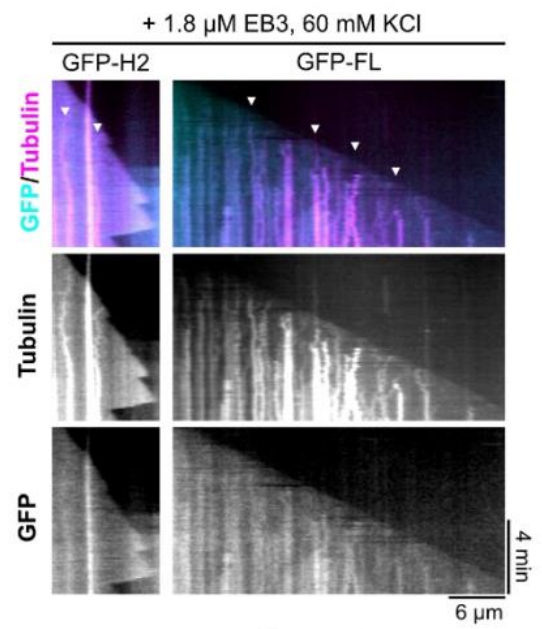

C

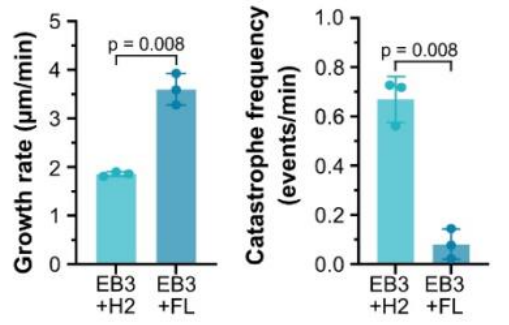

E
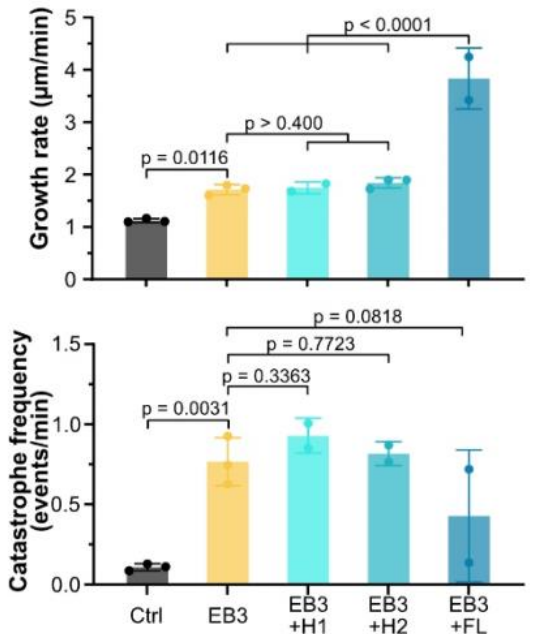

G

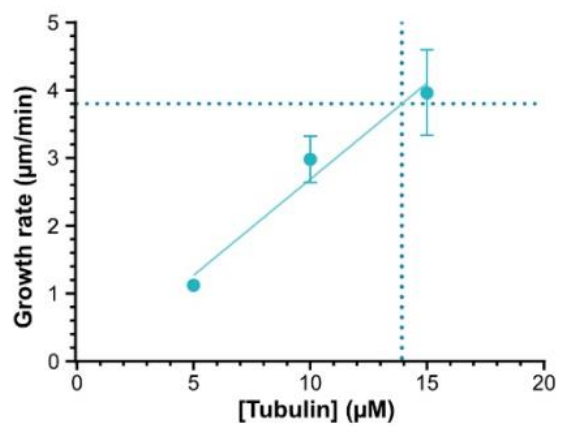


Figure 6: LLPS of +TIPs regulates microtubule dynamics through local tubulin condensation.

(A) Representative time-lapse TIRF images of GFP-FL-CLIP (50 nM) with Atto-565-tubulin (5 $\mu \mathrm{M})$ in the presence of unlabeled EB3 $(1.8 \mu \mathrm{M})$ and low salt $(60 \mathrm{mM} \mathrm{KCl})$ imaging buffer (see materials and methods). Time denoted in minutes: seconds; scale bar: $20 \mu \mathrm{m}$. Insets are enlargements of the area indicated by the dashed box. (B) Representative microtubule kymographs with $\mathrm{H} 2$ or FL-CLIP each at $50 \mathrm{nM}$ in the presence of EB3 $(1.8 \mu \mathrm{M})$ and tubulin $(5 \mu \mathrm{M})$ in low salt imaging buffer. White arrowheads denote tubulin/CLIP (right panel) and tubulin/H2 (left panel) co-condensation on the microtubule shaft. (C) Quantification of microtubule growth rate (left) and catastrophe frequency (right) in the presence of EB3/H2-networks and EB3/FL-CLIP droplets in low salt buffer $(60 \mathrm{mM} \mathrm{KCl})$. Mean with SD of three individual experiments with the following number of analyzed microtubules: EB3/H2 - 29; EB3/FL-CLIP - 59. Statistics: paired t-test. (D) Representative microtubule kymographs of denoted +TIP-networks in high salt buffer (60 mM KCl and $85 \mathrm{mM} \mathrm{K}$-acetate, see materials and methods). Note that tip-tracking efficiency (GFP channel) is weaker at $5 \mu \mathrm{M}$ tubulin than at higher tubulin concentrations (Figure $6 \mathrm{G}$ ). (E) Microtubule growth rate (top) and catastrophe frequency (bottom) in presence of denoted proteins in high salt buffer. Note that in high salt experiments, the catastrophe-inhibiting activity of EB3/FL-CLIP + TIP-droplets showed fluctuations between replicates. Both graphs show mean plus SD of three individual experiments (except for EB3/FL-CLIP, two experiments) with the following number of analyzed microtubules: Control - 48; EB3 - 31; EB3/H1 - 28; EB3/H2 - 28; EB3/FLCLIP - 41. Statistics: One-way ANOVA Fisher's LSD test. (F) Representative microtubule kymographs in the presence of GFP-H2 $(50 \mathrm{nM})$ and EB3 $(1.8 \mu \mathrm{M})$ grown at the denoted tubulin concentrations in high salt buffer. (G) Microtubule growth rate from experiments in G. Mean with SD from three individual experiments. Solid cyan line shows linear regression curve fit. Dashed blue line indicates concentration of tubulin at which microtubule in presence of $\mathrm{EB} 3 / \mathrm{H} 2$ networks grow at $3.8 \mu \mathrm{m} / \mathrm{min}$ (the speed achieved by EB3/FL-CLIP-droplets at $5 \mu \mathrm{M}$ tubulin; Figure $6 \mathrm{E}$ ). 


\section{Supplementary Figures S1-S6}
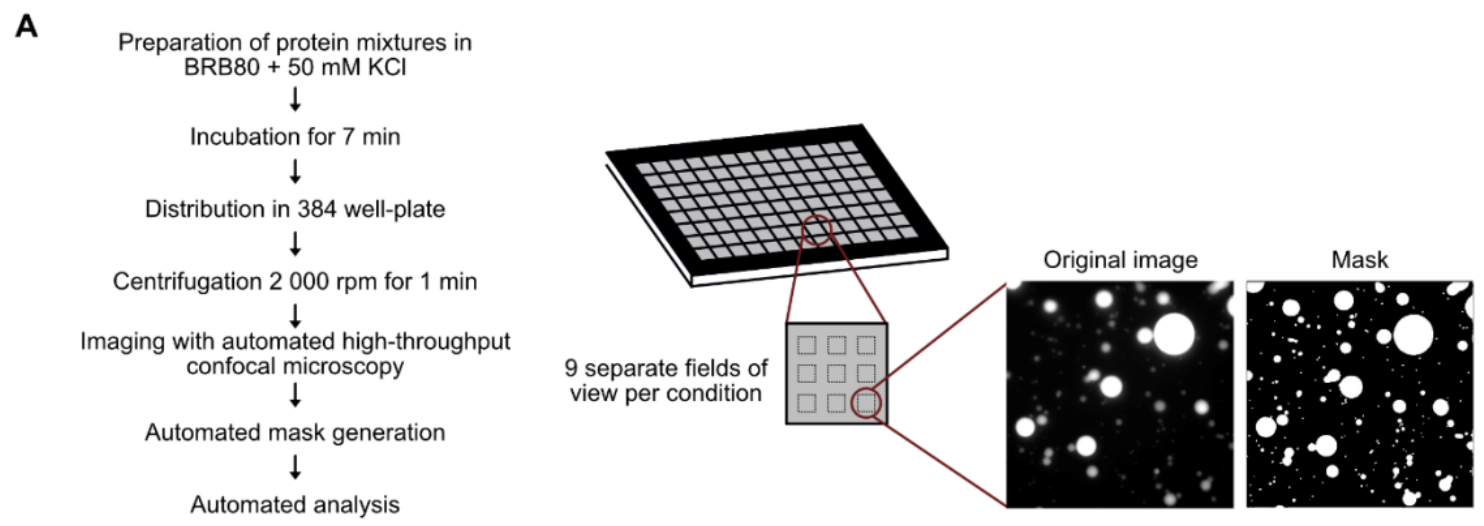

B

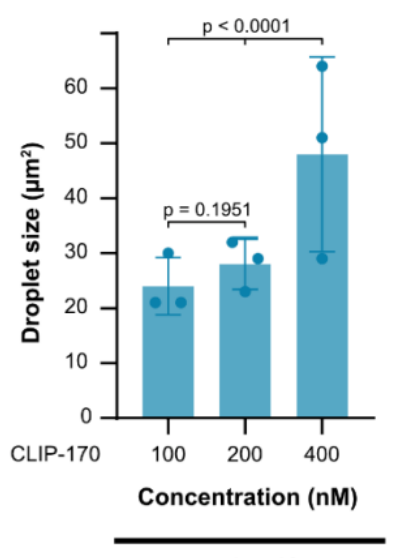

PEG $0 \%$
C
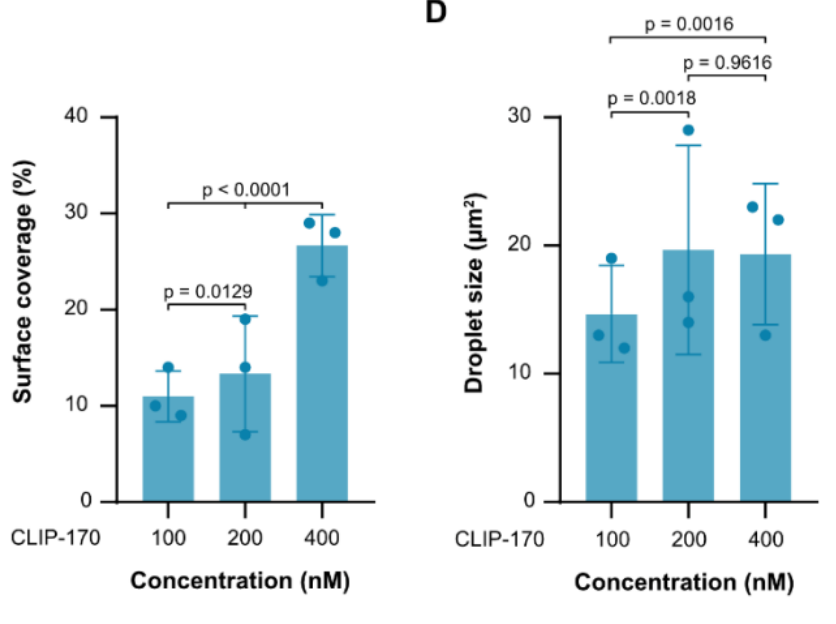

PEG $2 \%$

Figure S1: CLIP-170 droplet formation in vitro is sensitive to concentration and molecular crowding.

(A) Experimental outline for phase separation assays. For details, see materials and methods.

(B) Droplet size (area) for GFP-FL-CLIP condensates at 100, 200, or $400 \mathrm{nM}$ in the absence of PEG. (C) Coverslip surface coverage of GFP-FL-CLIP at the indicated concentrations in the presence of 2\% PEG. (D) Droplet size (area) of GFP-FL-CLIP condensates at 100, 200 and 400 $\mathrm{nM}$ in the presence of $2 \%$ PEG. All graphs show mean with SD from 3 independent experiments with a total of 27 fields of view. Statistics: two-tailed Student's $t$-test. 
A

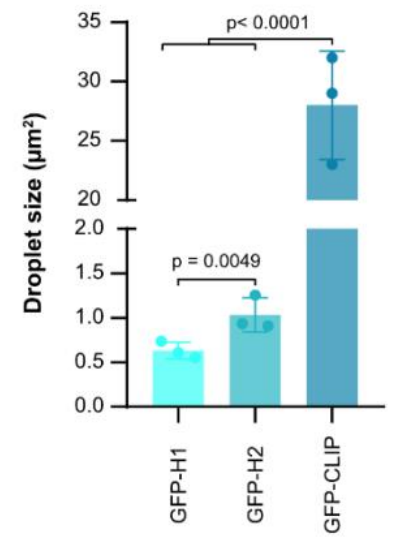

C

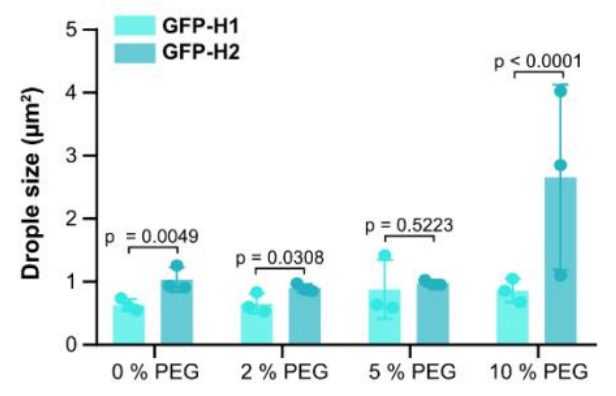

B
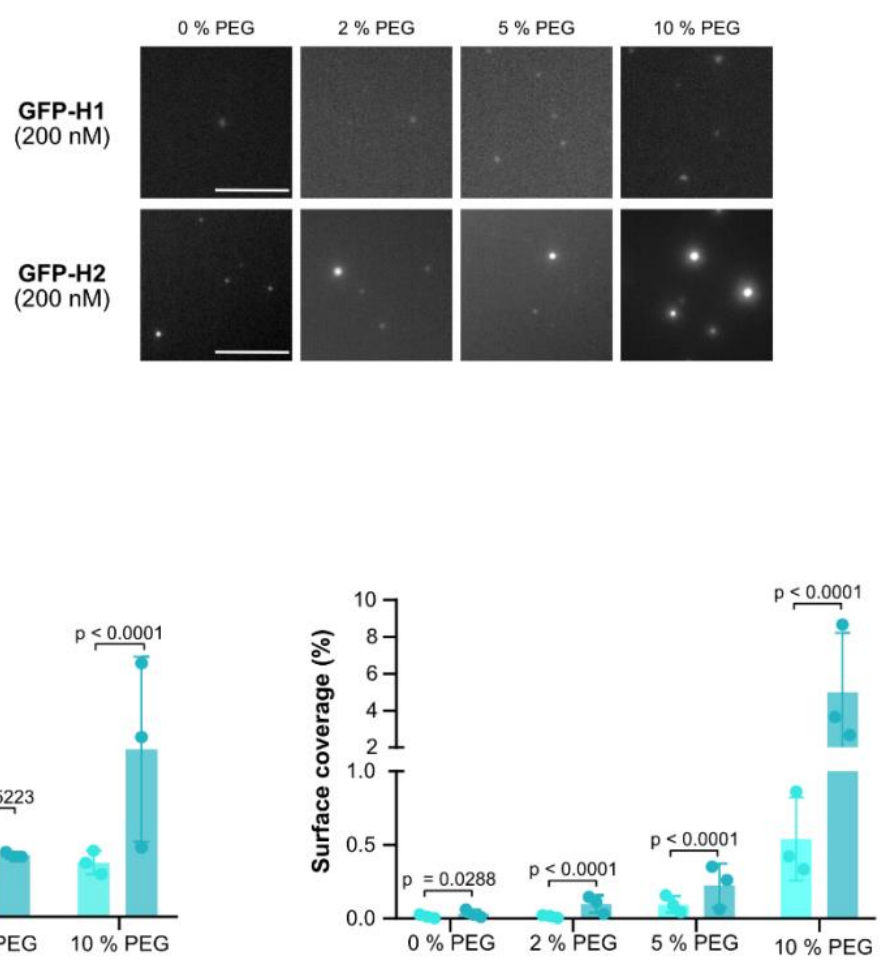

Figure S2: H2 only weakly undergo LLPS even under strong molecular crowding conditions. (A) Droplet size (area) of GFP-H2 (200 nM) and GFP-FL-CLIP (200 nM), and aggregate size of GFP-H1 $(200 \mathrm{nM})$ in the absence of PEG. Mean with SD from 3 independent experiments with a total of 27 fields of view. Statistics: one-way ANOVA test. (B) Representative fluorescence confocal images and quantification of purified GFP-H1 (200 nM) and GFP-H2 (200 nM) in the presence of 0,2, 5 and $10 \%$ PEG. Scale bar: $20 \mu \mathrm{m}$. (C) Droplet size (left graph) and surface coverage (right graph) of denoted proteins at indicated PEG concentrations. Mean with SD of from 27 fields of view from 3 independent experiments. Statistics: two-tailed Student's $t$-test.

A

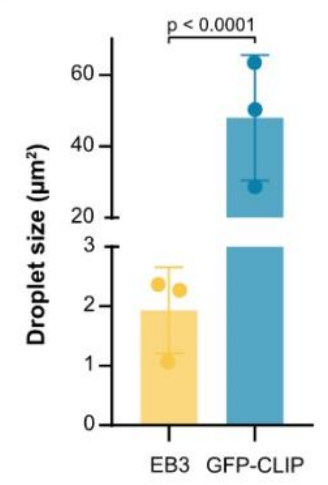

B

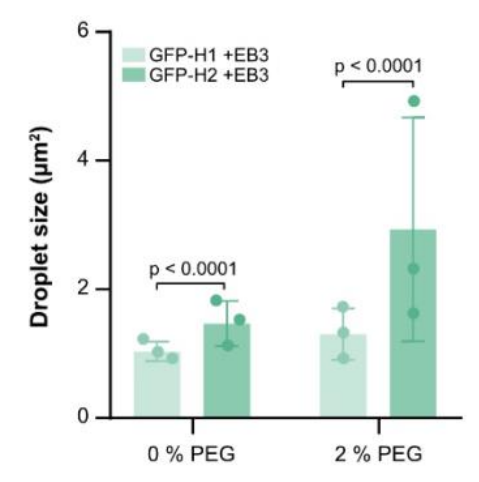


Figure S3: EB3/CLIP-170 phase separation is driven by the CLIP-170 C-terminal region in vitro.

(A) Droplet size (area) of unlabeled EB3 $(2.4 \mu \mathrm{M})$ and GFP-FL-CLIP (400 nM) at $0 \%$ PEG concentrations. Graph shows mean with SD of 3 independent experiments with a total of 27 fields of view. Statistics: two-tailed Student's $t$-test. (B) Condensate size (area) of GFP-H1 (200 nM) or GFP-H2 $(200 \mathrm{nM})$ in the presence of EB3 $(2.4 \mu \mathrm{M})$ in the absence or presence of $2 \%$ PEG. Mean with SD of 3 independent experiments with a total of 27 fields of view. Statistics: two-tailed Student's $t$-test 
A
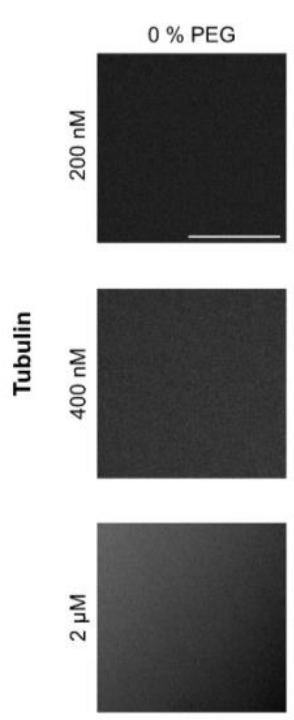

D
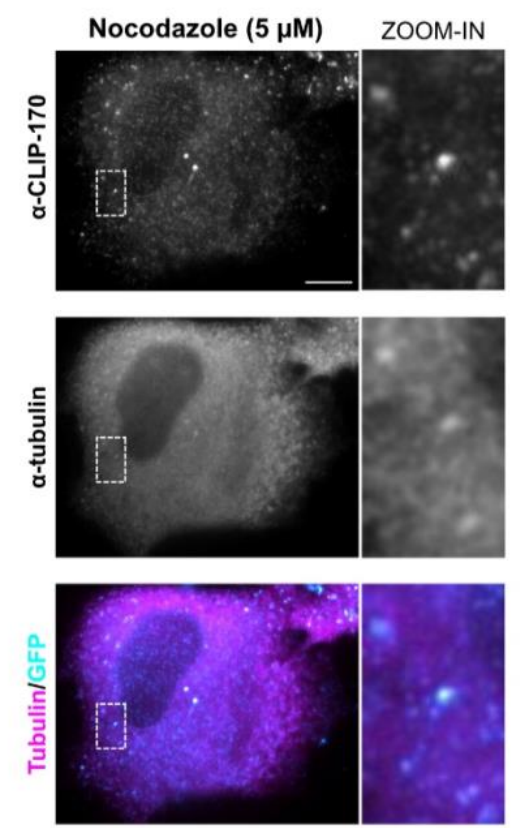

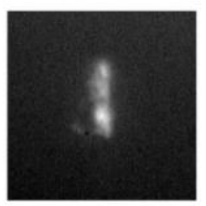

$2 \%$ PEG
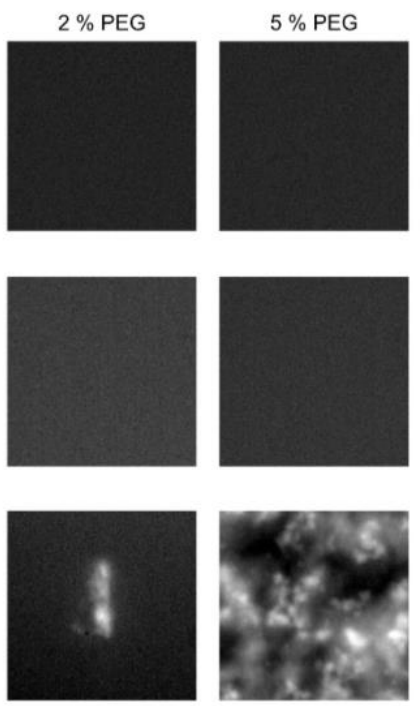

B

C
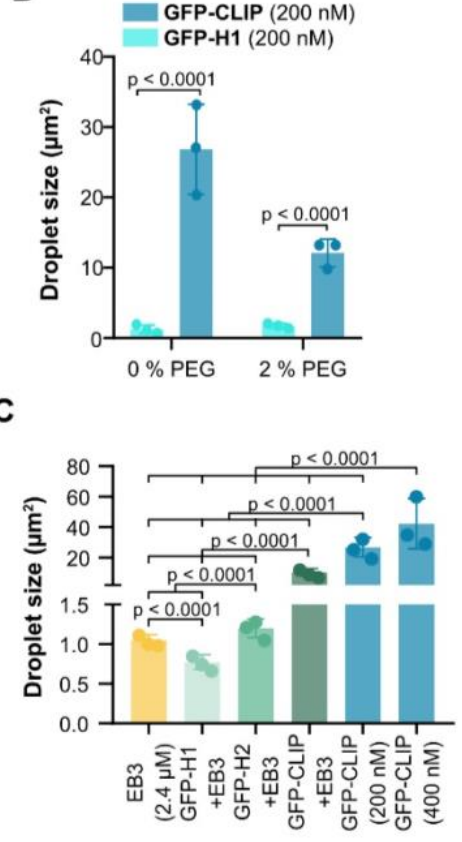

E Nocodazole $(5 \mu \mathrm{M})$

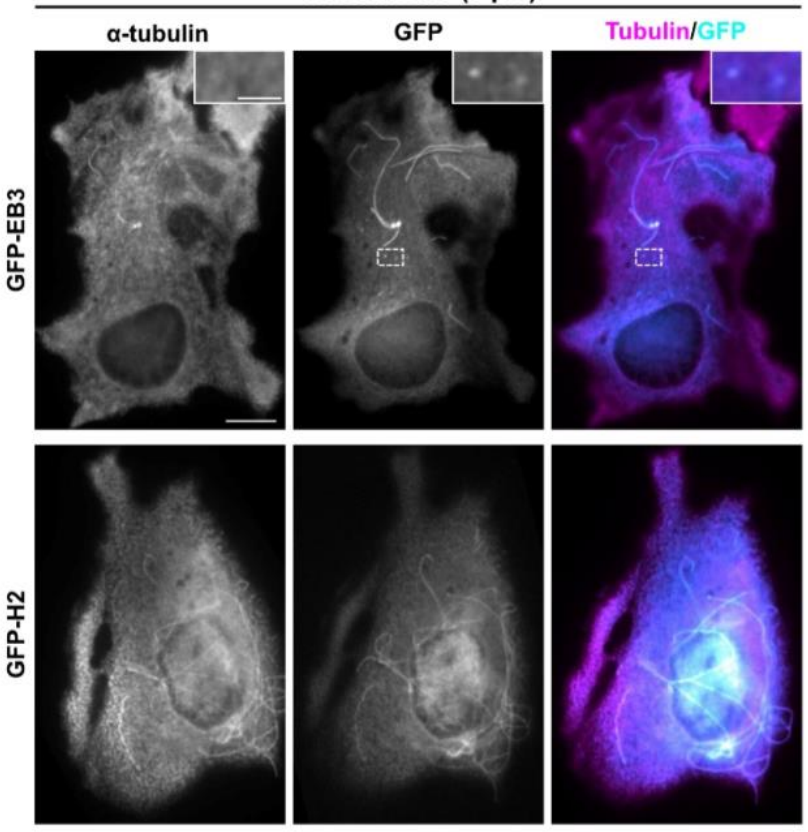

\section{Figure S4: CLIP-170 and EB3 form a tubulin-condensing network.}

(A) Representative fluorescence confocal images and quantification of purified Atto-565-tubulin at indicated concentrations in presence of 0,2 and $5 \%$ PEG. Note that at $2 \mu \mathrm{M}$ tubulin, PEG caused aggregation (but not condensate formation) of tubulin. Scale bar: $20 \mu \mathrm{m}$. (B) Droplet size of Atto-565-tubulin (400 nM) in the presence of GFP-H1 (200 nM) or GFP-FL-CLIP (200 nM) in the absence or presence of 2\% PEG. Mean with SD of from 27 fields of view from 3 independent experiments. Statistics: two-tailed Student's $t$-test (right graph). (C) Droplet size (area) of Atto565-tubulin (400 $\mathrm{nM})$ in the presence of purified EB3 $(2.4 \mu \mathrm{M})$, GFP-H1 (200 nM) and EB3 (2.4 
$\mu \mathrm{M})$, GFP-H2 (200 nM) and EB3 $(2.4 \mu \mathrm{M})$, GFP-FL-CLIP (200 nM) and EB3 (2.4 $\mu \mathrm{M})$, GFPFL-CLIP (200nM) alone, and GFP-FL-CLIP (400nM) alone. (D) Representative image of fixed RPE-1 WT cell stained for endogenous CLIP-170 and $\alpha$-tubulin after treatment with $5 \mu \mathrm{M}$ nocodazole for 1 hour. Inset is enlargement of region indicated by the dashed box. Scale bar: 10 $\mu \mathrm{m}$. (E) Representative images of fixed RPE-1 cells transfected with GFP-H2 (top panel) or GFPEB3 (bottom panel) treated with $5 \mu \mathrm{M}$ nocodazole for 1 hour and stained for tubulin. Scale bar: $10 \mu \mathrm{m}$.

A

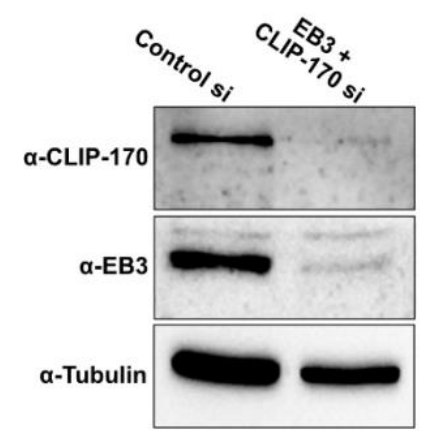

C

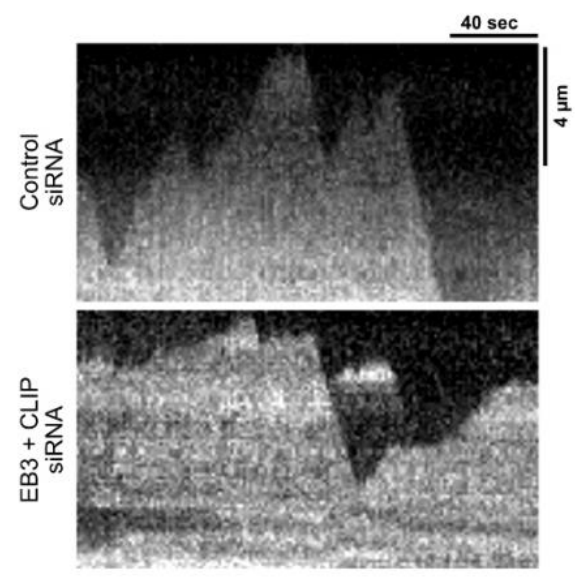

B

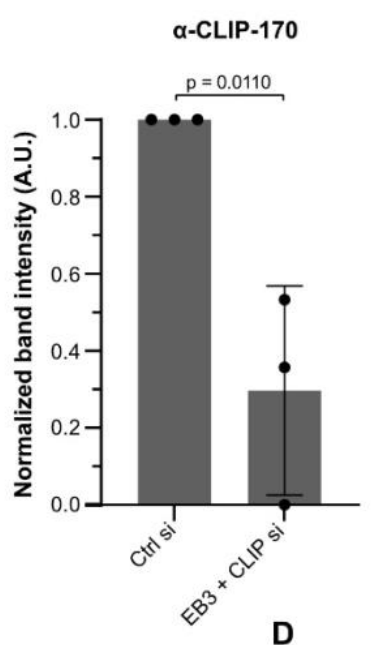

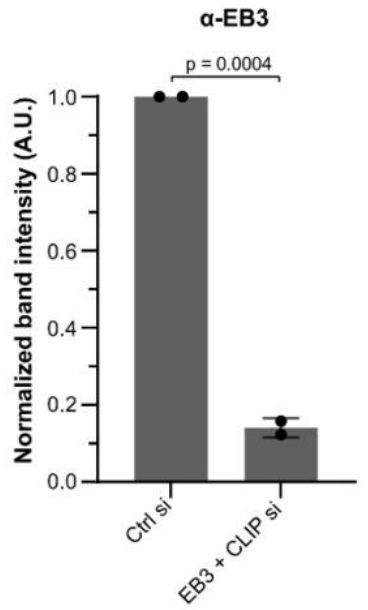

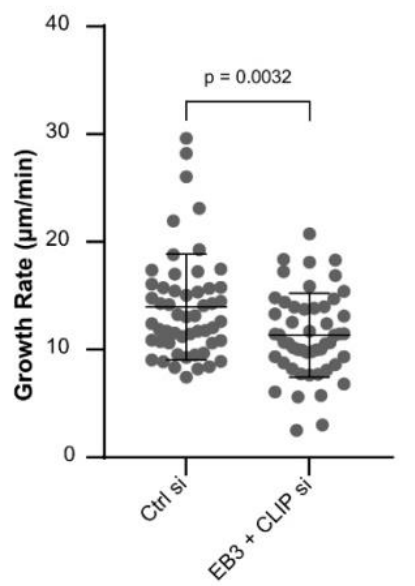

Figure S5: Depletion of EB3/CLIP-170 networks reduce microtubule growth rates in RPE-1 cells.

(A) Representative Western Blot of CRISPR/Cas9 knock-in GFP-Tubulin RPE-1 cells transfected with control siRNA or siRNA to CLIP-170 and EB3 simultaneously for 72 hours. (B) Quantification of western blot of CLIP-170 depletion (left) and EB3 depletion (right) in cells treated with either control or EB3 + CLIP-170 siRNAs. Graphs show mean with SD from $3(\alpha-$ CLIP-170) or 2 ( $\alpha$-EB3) individual experiments. Statistics: paired t-test.(C) Representative microtubule kymographs from CRISPR/Cas9 knock-in RPE-1-GFP-Tubulin cells transfected with either control (top) or EB3 + CLIP-170 (bottom) siRNAs for 72 hours. (D) Mean microtubule growth rate with SD from: Control - 53 microtubules from 29 cells; EB3 + CLIP siRNA - 51 
microtubules from 19 cells (4 independent experiments per condition). In graph, each dot represents a single microtubule. Statistics: paired t-test.
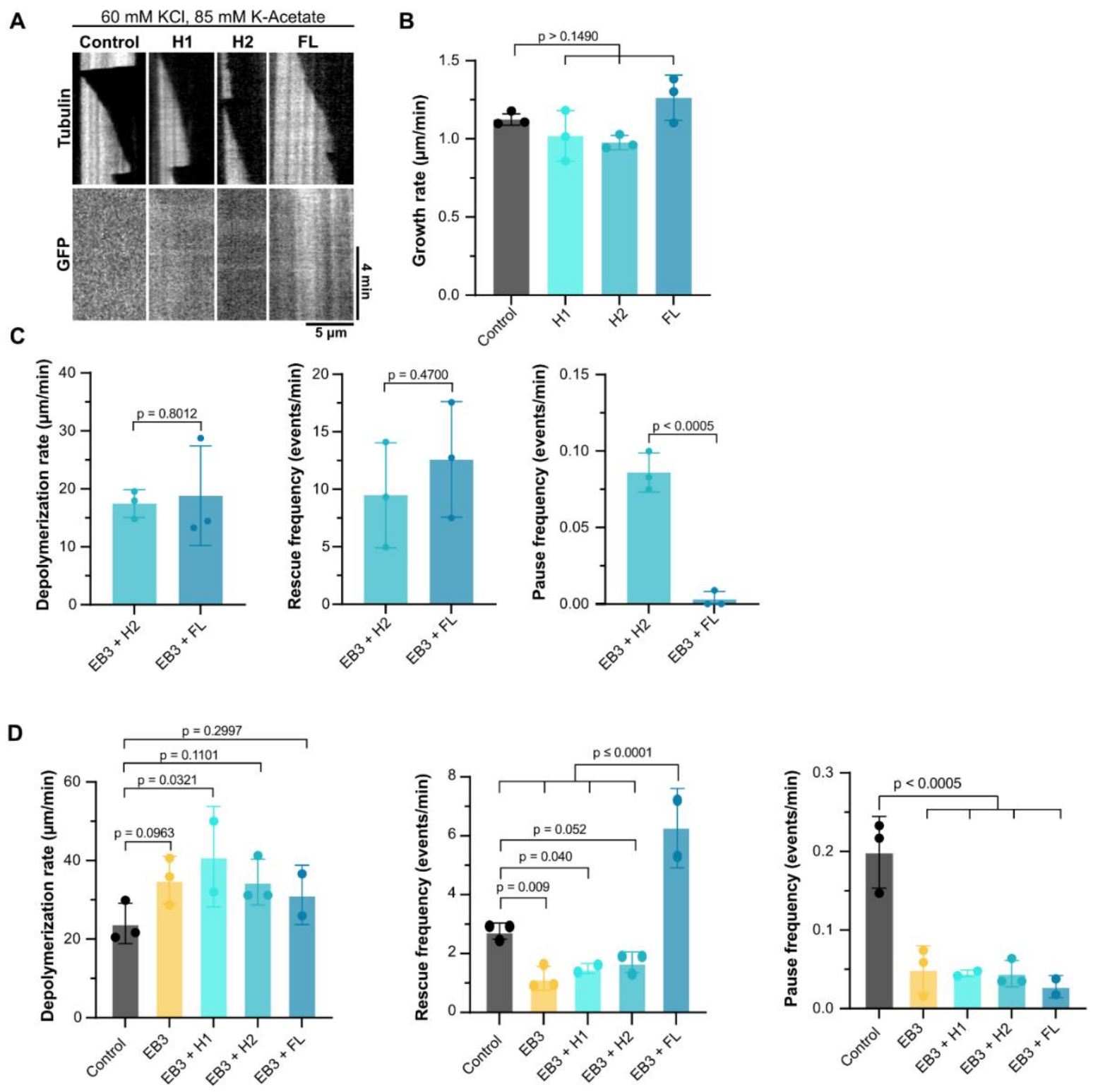

Figure S6: +TIP-droplets have stronger effects on microtubule dynamics than +TIPnetworks

(A) In vitro representative microtubule kymographs of control (no CLIP), 50 nM GFP-H1, or 50 nM GFP-H2 in high salt buffer. (B) Microtubule growth rate from experiments in figure A. Graphs show mean with SD of three individual experiments (dots) with the following numbers of microtubules analyzed: Control - 48; H1 - 42; H2 - 41. Statistics: one-way ANOVA Fisher's LSD test. (C) Microtubule dynamic parameters (depolymerization rate, left; rescue frequency, middle; and catastrophe frequency, right) for the denoted conditions in low salt buffer (corresponding assay to Figure 6C, see here for number of analyzed microtubules). Graphs show mean with SD of three individual experiments. Statistics: one-way ANOVA Fisher's LSD test. (D) Microtubule dynamic 
parameters (depolymerization rate, left; rescue frequency, middle; and catastrophe frequency, right) for the denoted conditions in high salt buffer (corresponding assay to Figure 6E, see here for number of analyzed microtubules). Graphs show mean with SD of three individual experiments, except for EB3 + FL-CLIP (two individual experiments). Statistics: one-way ANOVA Fisher's LSD test.

Movie 1: Representative RPE-1 cell overexpressing mCherry-CLIP imaged with TIRF microscopy. Inset highlights fusion of mCherry-CLIP droplets.

Movie 2: Representative RPE-1 cell overexpressing mCherry-CLIP imaged with TIRF microscopy. Inset highlights fission of mCherry-CLIP droplets.

Movie 3: Fluorescence recovery after photobleaching of a mCherry-CLIP droplet in a representative RPE-1 cell.

Movie 4: Fusion of two GFP-CLIP droplets $(1 \mu \mathrm{M})$ in vitro.

Movie 5: Fluorescence recovery after photobleaching of a purified GFP-CLIP droplet $(2 \mu \mathrm{M})$ in vitro.

Movie 6: Representative plus-end tracking behavior of overexpressed mcherry-H1 (cyan) in RPE-1 GFP-tubulin cells (magenta).

Movie 7: Representative plus-end tracking behavior of overexpressed mcherry-H2 (cyan) in RPE-1 GFP-tubulin cells (magenta).

Movie 8: Representative remnant formation resembling fission in GFP-CLIP overexpressing RPE-1 cells. Scale Bar: $2 \mu \mathrm{m}$.

Movie 9: Representative mCherry-EB3 expressing RPE-1 cell treated with 5\% 1,6-hexanediol (added at 00:30).

Movie 10: Representative in vitro microtubule dynamics assay of Atto-565 tubulin (magenta) polymerized in the presence of $50 \mathrm{nM}$ GFP-FL-CLIP (cyan) and 1.8 $\mu \mathrm{M}$ EB3 in low salt buffer.

Movie 11: Representative in vitro microtubule dynamics assay of Atto-565 tubulin (magenta) polymerized in the presence of $50 \mathrm{nM}$ GFP-FL-CLIP (cyan) and $1.8 \mu \mathrm{M}$ EB3 in high salt buffer. 


\section{Methods}

\section{Cell culture and treatments}

Parental RPE1 and CRISPR/Cas9 knock-in RPE1-GFP-tubulin cells (Andreu-Carbó et al., 2021) were cultured in high glucose Dulbecco's Modified Eagle's Medium F12 (DMEM, ThermoFisher, 113057) supplemented with $10 \%$ Fetal Bovine Serum (FBS, ThermoFisher, 10270106) and $1 \%$ penicillin-streptomycin (Gibco, 15140122) at $37^{\circ} \mathrm{C}$ with $5 \% \mathrm{CO} 2$. The cell lines were monthly checked for mycoplasma contamination.

For transient expression studies of exogenous CLIP and EB3, cells were transfected using the jetOPTIMUS transfection reagent (Polyplus) with $0.5 \mu \mathrm{g}$ DNA according to the manufacturer's instructions. Transfection media was replaced with fresh culture media 8 hours post-transfection, and cells were imaged 15-24h after transfection.

For microtubule dynamics studies, RPE1-GFP-Tubulin cells were transfected with siRNA using Lipofectamine RNAiMAX (Thermo Fisher) for 72 hours according to the manufacturer's instructions. For control experiments, cells were transfected with Allstars Negative Control siRNA (QIAGEN). For CLIP-170 + EB3 depletion experiments, cells were transfected with dual siRNA mixes targeting EB3 (Thermo Fisher, s22683) and CLIP-170 (Santa Cruz, 43281). Transfection media was replaced with fresh culture media 8 hours post-transfection, and cells were imaged 72 hours post-transfection.

To depolymerize the microtubule network, cells were treated with $5 \mu \mathrm{M}$ nocodazole (Sigma, M1404; diluted in culture medium) for 1 hour prior to fixation. For experiments using 1,6hexanediol (Sigma), cells were treated with 5\% 1,6-hexanediol (diluted in culture medium) for 10 minutes.

\section{Cloning}

The in-cell expression vector for mCherry-FL-CLIP170 was generated by excising GFP from a FL-CLIP170-GFP vector (a kind gift from Thomas Surrey) using AgeI/BsrG1 restriction sites and replacing it with mCherry containing AgeI/BsrG1 overhangs generated by PCR. From this vector, we generated mCherry-tagged H1- and H2-CLIP170 by PCR and reinsertion using the XhoI/KpnI restriction sites (N-terminal XhoI primer: 5'-CCGCTCGAGCTCAAGCTTCGATGAGTAT GCTGAAACCCAGCGGGCTGAA-3', C-terminal KpnI H1 primer: 5'-CGGGGTACCGTCGACTCAAGTGGTGCCCGAGATCTTGCGGGC-3', and C-terminal KpnI H2 primer: 5'-CGGGGTACCGTCGACTCATTTGTCAGCTTTGGTCTT TTCAAAGAGCAGGCTCTGTTC-3'). Protein purification vectors for H1- and H2-CLIP-GFP were generated by PCR from the FL-CLIP-170-GFP vector using a primer with an overhang for the Ndel restriction site as well as an N-terminal TEV protease site (5'GCGGCAGCCATATGGAAAACCTGTATTTCCAGGGAAGTGCCACCATGGTGAGCAAG GGCGAGGAGCTGTTCA-3'), and C-terminal primers specific to each CLIP truncation with overhangs corresponding to the Scal restriction site (H1: 5'-CCTTATCAAGTACTA GTGGTGCCCGAGATCTTGCGGGCGTAGCGGGAAG-3') (H2: 5'-CCTTATCAAGTACTT CATTTGTCAGCTTTGGTCTTTTCAAAGAGCAGGCTCTGTTCAAGC-3'), then cloned into 
an empty pET28a-6His vector (a kind gift from Natacha Olieric, Paul Scherrer Institute) using $\mathrm{NdeI} / \mathrm{ScaI}$ restriction sites.

A protein purification vector for mCherry-6His-EB3 was a kind gift from Natacha Olieric (Paul Scherrer Institute). From this vector, mCherry was excised using AgeI/BsrGI restriction sites to produce an untagged 6His-EB3 vector for purification.

For in-cell expression of EB3, mCherry-EB3 vectors were obtained from Addgene (Addgene plasmid 55037).

\section{Imaging}

\section{Microscope}

For in-cell studies and in vitro microtubule dynamics experiments, imaging was performed on an Axio Observer Inverted TIRF microscope (Zeiss, 3i) equipped with a Prime 95B ???BSI (Photometrics) using a 100X objective (Zeiss, Plan-Apochromat 100X/1.46 oil DIC (UV) VISIR). SlideBook 6 X 64 software (version 6.0.22) was employed to record time-lapse imaging. For in vitro microtubule dynamics and cell imaging, microscope stage conditions were controlled with the Chamlide Live Cell Instrument incubator $\left(37^{\circ} \mathrm{C}\right.$ for in vitro experiments, supplemented with 5 $\% \mathrm{CO}_{2}$ for live cell experiments).

\section{Microtubule dynamics}

For in-cell microtubule dynamic measurements images were taken every second for 3 minutes. Microtubules were tracked individually using the Freehand-Line tool in ImageJ (10-pixel width) and kymographs were built using the KymographBuilder plugin. Microtubule growth speeds were then calculated by manually tracing the slopes of kymographs using the Straight-Line tool in Image J and extracting the growth rate from the slopes using a custom-written code.

\section{Fluorescence recovery after photobleaching}

Fluorescence recovery after photobleaching (FRAP) experiments in cells were performed in square regions $(4 \mathrm{x} 4 \mu \mathrm{m})$ with a $656 \mathrm{~nm}$ laser at $20 \%$ intensity. The normalized fluorescence intensity was calculated using the formula $F(t)_{n o r m}=\frac{F(t)_{R O I}-F_{b c k}}{F(t)_{c t r l}-F_{b c k}} \times \frac{F(i)_{c t r l}-F_{b c k}}{F(i)_{R O I}-F_{b c k}}$ where $\mathrm{F}(\mathrm{t})_{\mathrm{ROI}}$ and $\mathrm{F}(\mathrm{t})_{\mathrm{ctrl}}$ are respectively the ROI and the control fluorescence intensity before the FRAP, $F_{b c k}$ the background fluorescence and $\mathrm{F}(\mathrm{i})_{\mathrm{ROI}}$ and $\mathrm{F}(\mathrm{i})_{\text {ctrl }}$ are respectively the ROI of the unbleached part of the condensate at one timepoint (i) (Day et al.,2012).

+ TIP-network analysis in 1,6-hexanediol-treated cells

For experiments in cells treated with 1,6-hexanediol, images were taken in a single z-plane every 10 seconds for 10 minutes. 1,6-Hexanediol (5\%) was added after one minute, and cells were only analyzed if they did not undergo any large-scale changes in morphology, as 1,6-hexanediol treatment has been noted to affect mammalian cell shape (Wheeler et al., 2016). For "pretreatment" time points, all in-focus +TIP-networks on the cell periphery were analyzed in the time frame 10 seconds prior to hexanediol addition using the Segmented-Line tool in ImageJ to obtain fluorescence intensity. For "post-treatment" time points, the same strategy was applied to the time frame 5 minutes after hexanediol addition. 


\section{Immunofluorescence}

15-24 hours post-transfection (or post-seeding for non-transfected cells), cells were fixed with $100 \%$ methanol for $5 \mathrm{~min}$ at $-20^{\circ} \mathrm{C}$ and then for $15 \mathrm{~min}$ with $3 \%$ paraformaldehyde at room temperature. Cells were then permeabilized for 10 minutes with $0.15 \%$ by volume Triton-X 100 (Sigma) in PBS followed by 10 minutes with 0.1\% Tween-20 (AppliChem) in PBS, washed thoroughly in a solution of $0.05 \%$ Tween-20 in PBS (hereafter referred to as PBS-T), and subsequently blocked with $2 \%$ bovine serum albumin (in PBS) for $1 \mathrm{~h}$. Post-blocking, cells were incubated overnight with antibodies targeting tubulin (Sigma T6199, DM1 $\alpha, 1: 1000$, mouse), EB1 (Millipore AB 6057, rabbit, 1:1000), EB3 (Santa Cruz Biotechnology sc-101475, KT36, rat, 1:200), or CLIP-170 (Santa Cruz Biotechnology sc-28325, F3, mouse, 1:500). Primary antibodies were diluted to the appropriate concentration in $2 \%$ bovine serum albumin in PBS. The following day, cells were subjected to three five-minute washes at room temperature in PBS-T, then subsequently incubated in secondary antibodies (Invitrogen, species-specific IgG conjugated to Alexa-647, 561, or 488 fluorophores) at room temperature for one hour. Cells were subjected to three additional PBS-T washes, and coverslips were mounted onto glass microscopy slides (Glass technology) using ProLong ${ }^{\mathrm{TM}}$ Diamont Antifade Mountant. Coverslips were sealed with nail polish and stored at $4^{\circ} \mathrm{C}$ until imaging.

\section{Tubulin purification from bovine brain and labelling}

Tubulin was purified from fresh bovine brain by two subsequent polymerization/depolymerization cycles as described previously (Andreu-Carbó et al., 2021). Tubulin labelling with biotin or ATTO-488, -565, -647 fluorophores was performed as described (Andreu-Carbó et al., 2021), and final labelling ratios to polymerize microtubules were $11 \%$ for ATTO-488 and $13 \%$ for ATTO565 tubulin.

\section{Protein purification}

For purification of EB3, E. coli BL21 (DE3) cells were transformed with 6-His-tagged EB3encoding plasmids and induced for expression overnight with $1 \mathrm{mM}$ IPTG at $20^{\circ} \mathrm{C}$ under rotation at $200 \mathrm{rpm}$. All following steps were performed at $4^{\circ} \mathrm{C}$. The morning after induction, cells were lysed by in lysis buffer $(20 \mathrm{mM}$ Tris $\mathrm{pH} 7.5,300 \mathrm{mM} \mathrm{NaCl})$ supplemented with $1 \%$ Triton-X 100 and protease inhibitors cocktail tablets (Roche) and sonicated. Cell debris were then cleared by ultracentrifugation. The cleared lysate was subsequently loaded onto a pre-equilibrated HisTrap column (GE Healthcare 1mL HisTrap column) using an ÄKTA Pure Protein Purification System (GE Healthcare). After washing the column in lysis buffer, elution buffer (20 mM Tris pH 7.5, 300 $\mathrm{mM} \mathrm{NaCl}, 1 \mathrm{M}$ imidazole) was applied to the column in a $1 \%$ gradient. Eluted protein fractions were pooled and concentrated using Amicon 30K Centrifugal filters (Millipore). The concentrated, cleared protein was subjected to size-exclusion chromatography using a HiLoad 16/600 Superdex column (GE Healthcare) in lysis buffer. Protein-containing fractions were harvested, pooled, and concentrated. Protein was supplemented with $20 \%$ glycerol, aliquoted, snap-frozen and stored at $80^{\circ} \mathrm{C}$. 
H1-CLIP-GFP and H2-CLIP-GFP were purified using the same scheme as EB3, with the following differences: (1) the lysis buffer was $50 \mathrm{mM}$ potassium phosphate $\mathrm{pH} 7.5,500 \mathrm{mM} \mathrm{NaCl}, 1 \mathrm{mM}$ $\mathrm{MgCl}_{2}$, and $1 \mathrm{mM} \beta$-mercaptoethanol. (2) Between applying protein to the HisTrap column and elution, the column was washed with lysis buffer supplemented with $8 \mathrm{mM}$ Imidazole. (3) H1 - and H2-CLIP were eluted from the HisTrap column using lysis buffer $+300 \mathrm{mM}$ Imidazole, and protein-containing fractions were subjected to tobacco etch virus (TEV) protease treatment overnight to remove His tags prior to size-exclusion chromatography.

FL-CLIP170-GFP was purified from insect cells as described previously (Telley et al., 2011). A plasmid encoding FL-CLIP170-GFP in pFasBacHTa (a kind gift from Thomas Surrey) was used to generate Baculovirus, which was subsequently used to infect Sf9 cells. Cells were harvested and lysed with lysis buffer (30 mM HEPES pH 7.4, $400 \mathrm{mM} \mathrm{KCl,} 20 \mathrm{mM}$ Arginine, $20 \mathrm{mM}$ potassium-glutamate, $0.01 \%$ Birj35, $2 \mathrm{mM} \mathrm{MgCl}$, $10 \mathrm{mM} \beta$-mercaptoethanol) supplemented with $20 \mathrm{mM}$ imidazole and protease inhibitor tablets (Roche) using dounce homogenization. Cell debris were cleared by ultracentrifugation, and cleared lysate was loaded onto a Histrap column (GE Healthcare). The column was washed with lysis buffer supplemented with $50 \mathrm{mM}$ imidazole, then eluted with lysis buffer supplemented with $300 \mathrm{mM}$ imidazole. Protein-containing fractions were pooled and further cleared by a second centrifugation, then subjected to size-exclusion chromatography using a HiLoad 16/600 Superdex column (GE Healthcare) in lysis buffer (lacking Birj35). Only proteins coming off with a clean, single peak profile from the Superdex column were used. Protein-containing fractions were pooled and concentrated and used immediately (maximal $5 \mathrm{hrs}$ after purification), as FL-CLIP was prone to degradation and loss of activity after freezing as previously noted (Telley et al., 2011). For all purified proteins, protein concentration was measured by Bradford assay.

\section{Phase separation assay}

For in vitro phase separation assays, proteins were diluted to the appropriate concentration in BRB80 supplemented with $50 \mathrm{mM}$ potassium chloride and PEG 4000 (0, 2, 5 or $10 \%$ by weight) in Eppendorf tubes. After thorough mixing, reactions were transferred to 384-well plates (Falcon) and incubated for $7 \mathrm{~min}$. The plate was then centrifuged at $2000 \mathrm{rpm}$ for $1 \mathrm{~min}$ to sediment proteins in the dense phase on the well bottoms. Images were acquired using a confocal automated microscopy (Molecular Device) with a 60X dry objective. For each reaction, 9 times 2048 x 2048 px fields of view were acquired with one focal plane (Figure S1A). Automated analysis was performed using MetaXpress Custom Module editor software. From fluorescence intensity, masks were generated to differentiate condensates from the background and the area sum was calculated together with the condensate versus background fluorescence intensity.

\section{Coverslip treatment and Flow chamber preparation}

For in vitro microtubule dynamics studies, slides and coverslips were cleaned by two successive 30 -minute sonication cycles in $1 \mathrm{M} \mathrm{NaOH}$ followed by $96 \%$ ethanol with thorough rinsing in bidistilled water between each step. After drying, slides and coverslips were plasma treated 
(Electronic Diener, Plasma surface technology) and subsequently incubated for 48 hours with triethoxy-silane-PEG (Creative PEGWorks) or a 1:5 mix of tri-ethoxy-silane-PEG-biotin: tri-ethoxysilane-PEG (final concentration $1 \mathrm{mg} / \mathrm{ml}$ ) in $96 \%$ ethanol and $0.02 \% \mathrm{HCl}$, with gentle agitation at room temperature. Slides and coverslips were then washed in ethanol (96\%) followed by thorough washing in bi-distilled water, then dried with an air gun and stored at $4^{\circ} \mathrm{C}$. Flow chambers were prepared by affixing a silane-PEG-biotin coverslip to a silane-PEG slide using double-sided tape.

\section{Microtubule dynamics assays in vitro}

Microtubule seeds were prepared at a final concentration of $10 \mu \mathrm{M}$ tubulin (20\% ATTO-647labelled tubulin and $80 \%$ biotinylated tubulin) in BRB80 supplemented with $0.5 \mathrm{mM}$ GMPCPP (Jena Bioscience) for 45 minutes at $37^{\circ} \mathrm{C}$. Seeds were incubated with $1 \mu \mathrm{M}$ Paclitaxel (Sigma) for 45 minutes at $37^{\circ} \mathrm{C}$, centrifuged $\left(50,000 \mathrm{rpm}\right.$ at $37^{\circ} \mathrm{C}$ for $\left.15 \mathrm{~min}\right)$, resuspended in $\mathrm{BRB} 80$ supplemented with $1 \mu \mathrm{M}$ Paclitaxel and $0.5 \mathrm{mM}$ GMPCPP, aliquoted and subsequently stored in liquid nitrogen.

Flow chambers were prepared by injecting subsequently $50 \mu \mathrm{g} / \mathrm{mL}$ neutravidin (ThermoFisher), BRB80, and microtubule seeds, then subsequently washing out unattached seeds with BRB80. Reaction buffer containing Atto-565 labelled-tubulin (1:5 ratio labelled to unlabelled; $5 \mu \mathrm{M}$ for all assays except for Figure 6G-H) in BRB80 supplemented with an anti-bleaching buffer [10 mM DTT, $0.3 \mathrm{mg} / \mathrm{mL}$ glucose, $0.1 \mathrm{mg} / \mathrm{mL}$ glucose oxidase, $0.02 \mathrm{mg} / \mathrm{mL}$ catalase, $0.125 \%$ methyl cellulose (1500 cP, Sigma), $1 \mathrm{mM} \mathrm{GTP]} \mathrm{was} \mathrm{subsequently} \mathrm{injected,} \mathrm{and} \mathrm{chambers} \mathrm{were} \mathrm{sealed}$ with silicon grease and immediately imaged. For "low salt" assays (Figure 6B-D), the reaction buffer was supplemented with $60 \mathrm{mM}$ potassium chloride. For "high salt" assays (Figure 6E-H), the reaction buffer was supplemented with $60 \mathrm{mM}$ potassium chloride and $85 \mathrm{mM}$ potassium acetate as described previously (Telley et al., 2011).

For assays involving recombinant EB3 and H1- or H2-CLIP-170, purified proteins were flashthawed and spun at 50,000 rpm in a TLA-100 centrifuge at $4^{\circ} \mathrm{C}$ for 15 minutes to remove any large aggregates. Proteins were diluted into BRB80 immediately prior to their usage, and further diluted to the appropriate concentration in reaction buffer. Assays involving FL-CLIP-170 were carried out as noted above, but within the first 5 hours post-purification as FL-CLIP-170 activity is poorly preserved after freezing (Telley et al., 2011).

\section{SDS-PAGE and Western blot}

Cell lysates were boiled run on SDS-PAGE gels (10\% acrylamide) and subsequently transferred to a nitrocellulose membrane using an iBLOT 2 Gel Transfer Device (ThermoFisher Scientific, IB21001). Nitrocellulose membranes were blocked for $1 \mathrm{~h}$ with $5 \%$ dried milk resuspended in TBS-Tween $1 \%$, then incubated over-night with primary antibodies: anti-beta-tubulin (Sigma, T6074, 1:1000 dilution) anti-EB3 (ATLAS anti-MAPRE3, HPA-009263, 1:500 dilution), or antiCLIP-170 (Santa Cruz Biotechnology, SC-28325, 1:1000 dilution). The following day, unbound antibodies were washed off with TBS-Tween $1 \%$, and membranes were incubated with secondary 
antibodies conjugated to horseradish peroxidase (anti-mouse or anti-rabbit; GE Healthcare 17097199 and 16951542, 1:5000 dilution) for 1 hour at room temperature. Following secondary antibody incubation, membranes were washed extensively with TBS-Tween $1 \%$ and imaged using an ECL Western blotting detection kit (Advansta) and with Fusion Solo Vilber Lourmat camera (Witec ag).

\section{Statistical analysis}

Statistical analyses were carried out using GraphPad Prism software v9 as described in figure legends. Unless otherwise noted, analyses were carried out between experimental means using one-way ANOVA Fisher's LSD test, or two-tailed Student's t-test. P-values less than 0.05 were considered statistically significant.

\section{References}

Akhmanova, A., \& Steinmetz, M. O. (2010). Microtubule +TIPs at a glance. J Cell Sci, 123(Pt 20), 3415-3419. https://doi.org/10.1242/jcs.062414

Akhmanova, A., \& Steinmetz, M. O. (2015). Control of microtubule organization and dynamics: two ends in the limelight. Nat Rev Mol Cell Biol, 16(12), 711-726.

https://doi.org/10.1038/nrm4084

Alberti, S., \& Dormann, D. (2019). Liquid-Liquid Phase Separation in Disease. Annual Review of Genetics, 53(1), 171-194. https://doi.org/10.1146/annurev-genet-112618-043527

Alberti, S., \& Dormann, D. (2019). Liquid-Liquid Phase Separation in Disease. Annual Review of Genetics, 53(1), 171-194. https://doi.org/10.1146/annurev-genet-112618-043527

Arnal, I., Heichette, C., Diamantopoulos, G. S., \& Chretien, D. (2004). CLIP-170/tubulin-curved oligomers coassemble at microtubule ends and promote rescues. Curr Biol, 14(23), 2086-2095. https://doi.org/10.1016/j.cub.2004.11.055

Bieling, P., Kandels-Lewis, S., Telley, I. A., van Dijk, J., Janke, C., \& Surrey, T. (2008). CLIP170 tracks growing microtubule ends by dynamically recognizing composite EB1/tubulinbinding sites. J Cell Biol, 183(7), 1223-1233. https://doi.org/10.1083/jcb.200809190

Boeynaems, S., Alberti, S., Fawzi, N. L., Mittag, T., Polymenidou, M., Rousseau, F., Schymkowitz, J., Shorter, J., Wolozin, B., Van Den Bosch, L., Tompa, P., \& Fuxreiter, M. (2018). Protein Phase Separation: A New Phase in Cell Biology. Trends Cell Biol, 28(6), 420 435. https://doi.org/10.1016/j.tcb.2018.02.004

Brouhard, G. J. (2015). Dynamic instability 30 years later: complexities in microtubule growth and catastrophe. Mol Biol Cell. 26(7), 1207-1210. https://doi.org/10.1091/mbc.E13-10-0594

Brouhard, G. J., \& Rice, L. M. (2018). Microtubule dynamics: an interplay of biochemistry and mechanics. Nat Rev Mol Cell Biol, 19(7), 451-463. https://doi.org/10.1038/s41580-018-0009-y 
Brouhard, G. J., Stear, J. H., Noetzel, T. L., Al-Bassam, J., Kinoshita, K., Harrison, S. C., Howard, J., \& Hyman, A. A. (2008). XMAP215 is a processive microtubule polymerase. Cell, 132(1), 79-88. https://doi.org/10.1016/j.cell.2007.11.043

Chen, J., Kholina, E., Szyk, A., Fedorov, V. A., Kovalenko, I., Gudimchuk, N., \& Roll-Mecak, A. (2021). alpha-tubulin tail modifications regulate microtubule stability through selective effector recruitment, not changes in intrinsic polymer dynamics. Dev Cell. https://doi.org/10.1016/j.devcel.2021.05.005

Diamantopoulos, G. S., Perez, F., Goodson, H. V., Batelier, G., Melki, R., Kreis, T. E., \& Rickard, J. E. (1999). Dynamic localization of CLIP-170 to microtubule plus ends is coupled to microtubule assembly. J Cell Biol, 144(1), 99-112. https://doi.org/10.1083/jcb.144.1.99

Dixit, R., Barnett, B., Lazarus, J. E., Tokito, M., Goldman, Y. E., \& Holzbaur, E. L. (2009). Microtubule plus-end tracking by CLIP-170 requires EB1. Proc Natl Acad Sci U S A, 106(2), 492-497. https://doi.org/10.1073/pnas.0807614106

Duellberg, C., Trokter, M., Jha, R., Sen, I., Steinmetz, M. O., \& Surrey, T. (2014).

Reconstitution of a hierarchical +TIP interaction network controlling microtubule end tracking of dynein. Nat Cell Biol, 16(8), 804-811. https://doi.org/10.1038/ncb2999

Galjart, N. (2010). Plus-end-tracking proteins and their interactions at microtubule ends. Curr Biol, 20(12), R528-537. https://doi.org/10.1016/j.cub.2010.05.022

Gard, D. L., \& Kirschner, M. W. (1987). A microtubule-associated protein from Xenopus eggs that specifically promotes assembly at the plus-end. J Cell Biol, 105(5), 2203-2215. https://doi.org/10.1083/jcb.105.5.2203

Goodson, H. V., Skube, S. B., Stalder, R., Valetti, C., Kreis, T. E., Morrison, E. E., \& Schroer, T. A. (2003). CLIP-170 interacts with dynactin complex and the APC-binding protein EB1 by different mechanisms. Cell Motil Cytoskeleton, 55(3), 156-173.

https://doi.org/10.1002/cm.10114

Gupta, K. K., Joyce, M. V., Slabbekoorn, A. R., Zhu, Z. C., Paulson, B. A., Boggess, B., \& Goodson, H. V. (2010). Probing interactions between CLIP-170, EB1, and microtubules. J Mol Biol, 395(5), 1049-1062. https://doi.org/10.1016/j.jmb.2009.11.014

Henrie, H., Bakhos-Douaihy, D., Cantaloube, I., Pilon, A., Talantikite, M., Stoppin-Mellet, V., Baillet, A., Pous, C., \& Benoit, B. (2020). Stress-induced phosphorylation of CLIP-170 by JNK promotes microtubule rescue. J Cell Biol, 219(7). https://doi.org/10.1083/jcb.201909093

Hernandez-Vega, A., Braun, M., Scharrel, L., Jahnel, M., Wegmann, S., Hyman, B. T., Alberti, S., Diez, S., \& Hyman, A. A. (2017). Local Nucleation of Microtubule Bundles through Tubulin Concentration into a Condensed Tau Phase. Cell Rep, 20(10), 2304-2312.

https://doi.org/10.1016/j.celrep.2017.08.042 
Howard, J., \& Hyman, A. A. (2009). Growth, fluctuation and switching at microtubule plus ends. Nat Rev Mol Cell Biol, 10(8), 569-574. https://doi.org/10.1038/nrm2713

Hyman AA, Weber CA, Jülicher F. Liquid-liquid phase separation in biology. Annu Rev Cell Dev Biol. 2014;30:39-58. https://doi.org/10.1146/annurev-cellbio-100913-013325

Jiang, H., Wang, S., Huang, Y., He, X., Cui, H., Zhu, X., \& Zheng, Y. (2015). Phase transition of spindle-associated protein regulate spindle apparatus assembly. Cell, 163(1), 108-122. https://doi.org/10.1016/j.cell.2015.08.010

Jiang, X., Ho, D. B. T., Mahe, K., Mia, J., Sepulveda, G., Antkowiak, M., Jiang, L., Yamada, S., \& Jao, L. E. (2021). Condensation of pericentrin proteins in human cells illuminates phase separation in centrosome assembly. J Cell Sci. https://doi.org/10.1242/jcs.258897

King, M. R., \& Petry, S. (2020). Phase separation of TPX2 enhances and spatially coordinates microtubule nucleation. Nat Commun, 11(1), 270. https://doi.org/10.1038/s41467-019-14087-0

Komarova, Y., De Groot, C. O., Grigoriev, I., Gouveia, S. M., Munteanu, E. L., Schober, J. M., Honnappa, S., Buey, R. M., Hoogenraad, C. C., Dogterom, M., Borisy, G. G., Steinmetz, M. O., \& Akhmanova, A. (2009). Mammalian end binding proteins control persistent microtubule growth. J Cell Biol, 184(5), 691-706. https://doi.org/10.1083/jcb.200807179

Komarova, Y., Lansbergen, G., Galjart, N., Grosveld, F., Borisy, G. G., \& Akhmanova, A. (2005). EB1 and EB3 control CLIP dissociation from the ends of growing microtubules. Mol Biol Cell, 16(11), 5334-5345. https://doi.org/10.1091/mbc.e05-07-0614

Komarova, Y. A., Akhmanova, A. S., Kojima, S., Galjart, N., \& Borisy, G. G. (2002).

Cytoplasmic linker proteins promote microtubule rescue in vivo. J Cell Biol, 159(4), 589-599. https://doi.org/10.1083/jcb.200208058

Kroschwald, S., Maharana, S., \& Simon, A. (2017). Hexanediol: a chemical probe to investigate the material properties of membrane-less compartments.

https://doi.org/10.19185/MATTERS.201702000010

Maan, R., Reese, L., Volkov, V. A., King, M. R., van der Sluis, E., Andrea., N., Evers, W., Jakobi, A. J., and Dogterom, M. (2021). Multivalent interactions facilitate motor-dependent protein accumulation at growing microtubule plus ends. Biorxiv. https://doi.org/10.1101/2021.09.14.460284

Meier., S. A., Farcas, A., Kumar, A., Ijavi, M., Bill., R. T., Stelling, J., Dufresne, E., Steinmetz, M. O., and Barral, Y. (2021). High interaction valency ensures cohesion and persistence of a microtubule +TIP body at the plus-end of a single specialized microtubule in yeast. Biorxiv. https://doi.org/10.1101/2021.09.13.460064 
Montenegro Gouveia, S., Leslie, K., Kapitein, L. C., Buey, R. M., Grigoriev, I., Wagenbach, M., Smal, I., Meijering, E., Hoogenraad, C. C., Wordeman, L., Steinmetz, M. O., \& Akhmanova, A. (2010). In vitro reconstitution of the functional interplay between MCAK and EB3 at microtubule plus ends. Curr Biol, 20(19), 1717-1722. https://doi.org/10.1016/j.cub.2010.08.020

Mustyatsa, V. V., Kostarev, A. V., Tvorogova, A. V., Ataullakhanov, F. I., Gudimchuk, N. B., \& Vorobjev, I. A. (2019). Fine structure and dynamics of EB3 binding zones on microtubules in fibroblast cells. Mol Biol Cell, 30(17), 2105-2114. https://doi.org/10.1091/mbc.E18-11-0723

Perez, F., Diamantopoulos, G. S., Stalder, R., \& Kreis, T. E. (1999). CLIP-170 highlights growing microtubule ends in vivo. Cell, 96(4), 517-527. https://doi.org/10.1016/s0092$\underline{8674(00) 80656-X}$

Pierre, P., Pepperkok, R., \& Kreis, T. E. (1994). Molecular characterization of two functional domains of CLIP-170 in vivo. J Cell Sci, 107 ( Pt 7), 1909-1920.

https://www.ncbi.nlm.nih.gov/pubmed/7983157

Pierre, P., Scheel, J., Rickard, J. E., \& Kreis, T. E. (1992). CLIP-170 links endocytic vesicles to microtubules. Cell, 70(6), 887-900. https://doi.org/10.1016/0092-8674(92)90240-d

Seetapun D, Castle BT, McIntyre AJ, Tran PT, Odde DJ. Estimating the microtubule GTP cap size in vivo. Curr Biol. 2012 Sep 25;22(18):1681-7. https://doi.org/10.1016/j.cub.2012.06.068

Shin Y, Brangwynne CP. Liquid phase condensation in cell physiology and disease. Science. 2017 Sep 22;357(6357):eaaf4382. https://doi.org/10.1126/science.aaf4382

Srayko, M., Kaya, A., Stamford, J., \& Hyman, A. A. (2005). Identification and characterization of factors required for microtubule growth and nucleation in the early $\mathrm{C}$. elegans embryo. Dev Cell, 9(2), 223-236. https://doi.org/10.1016/j.devcel.2005.07.003

Straube, A., \& Merdes, A. (2007). EB3 regulates microtubule dynamics at the cell cortex and is required for myoblast elongation and fusion. Curr Biol, 17(15), 1318-1325.

https://doi.org/10.1016/j.cub.2007.06.058

Telley, I. A., Bieling, P., \& Surrey, T. (2011). Reconstitution and quantification of dynamic microtubule end tracking in vitro using TIRF microscopy. Methods Mol Biol, 777, 127-145. https://doi.org/10.1007/978-1-61779-252-6_10

Vitre, B., Coquelle, F. M., Heichette, C., Garnier, C., Chretien, D., \& Arnal, I. (2008). EB1 regulates microtubule dynamics and tubulin sheet closure in vitro. Nat Cell Biol, 10(4), 415-421. https://doi.org/10.1038/ncb1703

Voter, W. A., O'Brien, E. T., \& Erickson, H. P. (1991). Dilution-induced disassembly of microtubules: relation to dynamic instability and the GTP cap. Cell Motil Cytoskeleton, 18(1), 55-62. https://doi.org/10.1002/cm.970180106 
Walker, R. A., O'Brien, E. T., Pryer, N. K., Soboeiro, M. F., Voter, W. A., Erickson, H. P., \& Salmon, E. D. (1988). Dynamic instability of individual microtubules analyzed by video light microscopy: rate constants and transition frequencies. J Cell Biol, 107(4), 1437-1448.

https://doi.org/10.1083/jcb.107.4.1437

Woodruff, J. B., Ferreira Gomes, B., Widlund, P. O., Mahamid, J., Honigmann, A., \& Hyman, A. A. (2017). The Centrosome Is a Selective Condensate that Nucleates Microtubules by Concentrating Tubulin. Cell, 169(6), 1066-1077 e1010.

https://doi.org/10.1016/j.cell.2017.05.028

Wu, Y.-F. O., Bryant, A. T., Nelson, N. T., Madey, A. G., Fernandes, G. F., \& Goodson, H. V. (2021). Overexpression of the microtubule-binding protein CLIP-170 induces a +TIP network superstructure consistent with a biomolecular condensate. bioRxiv, 2021.2001.2001.424687. https://doi.org/10.1101/2021.01.01.424687

Yang, C., Wu, J., de Heus, C., Grigoriev, I., Liv, N., Yao, Y., Smal, I., Meijering, E., Klumperman, J., Qi, R. Z., \& Akhmanova, A. (2017). EB1 and EB3 regulate microtubule minus end organization and Golgi morphology. J Cell Biol, 216(10), 3179-3198.

https://doi.org/10.1083/jcb.201701024

Zanic, M., Widlund, P. O., Hyman, A. A., \& Howard, J. (2013). Synergy between XMAP215 and EB1 increases microtubule growth rates to physiological levels. Nat Cell Biol, 15(6), 688693. https://doi.org/10.1038/ncb2744 